\title{
SLIM SEMIMODULAR LATTICES. II. A DESCRIPTION BY PATCHWORK SYSTEMS
}

\author{
GÁBOR CZÉDLI AND E. TAMÁS SCHMIDT
}

\begin{abstract}
Rectangular lattices are special planar semimodular lattices introduced by G. Grätzer and E. Knapp in 2009. By a patch lattice we mean a rectangular lattice whose weak corners are coatoms. As a sort of gluings, we introduce the concept of a patchwork system. We prove that every glued sum indecomposable planar semimodular lattice is a patchwork of its maximal patch lattice intervals "sewn together"; see Figure 3 for a first impression. For a modular planar lattice, our patchwork system coincides with the $S$-glued system introduced by C. Herrmann in 1973. Among planar semimodular lattices, patch lattices are characterized as the patchwork-irreducible ones. They are also characterized as the indecomposable ones with respect to the Hall-Dilworth gluing over chains; this fact gives another structure theorem for planar semimodular lattices since patch lattices are obtained from the four-element non-chain lattice by adding forks, introduced in our preceding paper.
\end{abstract}

\section{INTRODUCTION}

Rectangular lattices were introduced by Grätzer and Knapp [16]. Roughly speaking, a rectangular lattice is a planar semimodular lattice such that the contour of its natural diagram is a rectangle. The smallest rectangular lattice is the four-element Boolean lattice $\mathbf{2}^{2}$. If $L$ is a non-chain lattice such that each $x \in L \backslash\{0,1\}$ is incomparable with some element of $L$, then $L$ is glued sum indecomposable.

Let $L$ be a glued sum indecomposable planar distributive lattice. By the folklore of lattice theory, see Grätzer and Knapp [15], the diagram of $L$ can be decomposed into $\mathbf{2}^{2}$-intervals (that is, intervals isomorphic to $\mathbf{2}^{2}$ ), and for any two distinct $2^{2}$-intervals $I$ and $J$,

$$
I \cap J \text { is a chain, or } I \cap J=\emptyset .
$$

We will say that the $\mathbf{2}^{2}$-intervals of $L$ form a patchwork system $\boldsymbol{H}$ for $L$. This terminology is motivated by the everyday's life, where pieces of cloth of various colors and shape (but usually rectangular shape) sewn together form a so-called patchwork. Clearly, $\mathbf{2}^{2}$ is "patchwork-irreducible" since it is the smallest rectangular lattice.

$S$-glued systems were introduced by Herrmann [20] (not only for planar lattices). Let $M$ be a glued sum indecomposable planar modular lattice. Then the maximal atomistic (equivalently, complemented) intervals of $M$ are rectangular lattices of

Date: August 23, 2011.

1991 Mathematics Subject Classification. Primary 06C10 .

Key words and phrases. Slim lattice, semimodularity, planar lattice, patchwork.

This research was supported by the NFSR of Hungary (OTKA), grant numbers K77432 and K83219 
length two, and they form an $S$-glued system $\mathcal{H}=\mathcal{H}^{\text {Herrm }}(M)$. Clearly, (1.1) holds again, and the intervals we consider are "patchwork-irreducible" again since they have no proper rectangular subinterval. (For more details see Lemma 3.8 later.) Hence $\mathcal{H}^{\text {Herrm }}(M)$ in this case we will also be called a patchwork system.

Motivated by the above ideas, our goal is the develop a theory of patchwork systems for all planar semimodular lattices; see Figures 2 and 3 for a first impression. Rectangular lattices whose weak corners are coatoms will be called patch lattices since they will turn out to be exactly the patchwork-irreducible planar semimodular lattices. Surprisingly, patch lattices will also be characterized as semimodular lattices indecomposable with respect to the Hall-Dilworth gluing over chains. Hence patch lattices give rise to two structure theorems for planar semimodular lattices: one of them is based on patchwork systems (see Theorem 3.6 for details), while the other one (see Corollary 3.5) is based on the Hall-Dilworth gluing over chains. Of course, the value of these theorems depends on how the building stones, the patch lattices, can be described. We will extract a constructive visual structure theorem for patch lattices from [7], see Theorem 3.4(vii) or, for a first impression, see Figure 2.

The structure theorem in [7] for planar semimodular lattices (the conjunction of Propositions 2.1 and 2.2 here) needed three constructive steps to obtain all planar semimodular lattices. These constructive steps were quite easy but not widely known yet. Namely, we added "forks", deleted "corners", and added "eyes". One of our new structure theorems, Corollary 3.5, is based on the classical Hall-Dilworth gluing over chains plus only on two recently introduced steps (adding forks and adding eyes).

Outline. Based mainly on Kelly and Rival [23], Grätzer and Knapp [15] and [16], and [6] and [7], Section 2 surveys those known concepts and facts on planar semimodular lattices that we need in the paper. Among all planar semimodular lattices, the so-called slim ones play a distinguished role; Section 2 explains why.

Section 3 gives the most important new concepts and the main results. In particular, it formulates the results mentioned in (this) Section 1 and in the Abstract.

Many of the concepts we deal with depend on the planar diagram chosen, at least formally. This motivates the study of these diagrams and some related questions in Section 4. Lemmas 4.1, 4.3, 4.7, 4.8 and 4.9 are of independent interest. For example, Lemma 4.7 asserts that the diagram of a slim semimodular lattice is uniquely determined in some sense, while Lemma 4.9 says that rectangularity is independent from the diagram chosen.

Section 5 is devoted to elements and rectangular intervals of $L$ versus planar diagrams of $L$; Lemmas 5.3 and 5.4 are worth mentioning here.

Section 6 presents a lot of properties of a planar semimodular lattice $L$ that depend only on a slim semimodular lattice "canonically" derived from $L$.

Section 7 proves Proposition 3.2, asserting that all properties of $L$ that are really important from our perspective are independent from the diagram of $L$.

Section 8 formulates and proves several properties of patchwork-indecomposable lattices; these properties are consequences of the main results stated in Section 3.

As further consequences of the main results of Section 3, Section 9 proves some properties of patchwork-indecomposable intervals of planar semimodular lattices.

Based on the auxiliary statements of all other sections, Section 10 completes the paper by proving the main results formulated in Section 3. 


\section{Preliminaries}

We aim at planar semimodular lattices. However, most questions of the planar case are easily deductible from the slim one; for example, see Proposition 2.1 and Lemma 6.1 later. This fact and the number "I" in [7] explains that the adjective "slim" rather than "planar" occurs in the title of the present paper.

Slimness. These years are witnessing an increasing role of slimness (to be defined later) in Lattice Theory, see Grätzer and Knapp [15] and [17], and [2], [4], [5], [6], [7], [8], [9] and [24]. The importance of slim lattices is explained by three facts.

Firstly, slim semimodular lattices are, up to dual isomorphism, exactly the lattices that we obtain from two (finite) composition series of a group by forming all intersections of their members. This explains that soon after Grätzer and Nation [18] improved the classical Jordan-Hölder theorem, see Hölder [21] and Jordan [22], we could add a uniqueness part to this theorem in [6], and even the number how many ways two composition series can intersect has recently been determined in [9].

Secondly, slim lattices are planar by [6, Lemma 6], and planar semimodular lattices play an intensive role in various results on the finite congruence lattice representation problem. An overview is given in Grätzer [14] while [3], [24], and Grätzer and Knapp [16] and [17] represent some recent developments.

Thirdly, each planar semimodular lattice is obtained from a unique slim semimodular lattice in a very simple way, see Proposition 2.1 and Lemma 4.1 later. This explains that although many of the proofs deal with slim semimodular lattices, our theorems hold for all planar semimodular lattices.

Basic concepts. All lattices occurring in this paper are assumed to be finite, even if this is not mentioned all the time. In particular, if a lattice is slim or planar, then it is finite by definition. In most of the cases, our lattices are assumed to have at least four elements. The systematic study of slim semimodular lattices started in Grätzer an Knapp [15]. Recall that a lattice $L$ is called (upper) semimodular, if $b \vee c$ covers or equals $a \vee c$ for all $a, b, c \in L$ with $a \prec b$. Because of their links to combinatorics and geometry, the study of these lattices is an important branch of Lattice Theory; see Stern [25] and [5] for surveys. By a slim lattice we mean a finite lattice $M$ such that the order $\operatorname{Ji}(M)$ of (non-zero) join-irreducible elements of $M$ contains no three-element antichain. (Orders are also called partially ordered sets or posets.) Equivalently, see Dilworth [11], Grätzer and Knapp [15] or [7], a finite lattice $M$ is slim iff $\operatorname{Ji}(M)$ is the union of two chains. As already mentioned, slim lattices are planar. A straightforward but extremely useful property of slim lattices, see [7, Lemma 2], is that

$$
\text { each element of a slim lattice has at most two covers. }
$$

Another pleasant property is that

$$
\text { every interval of a slim lattice is slim; }
$$

this follows from the fact that $\{a \vee x: x \in \operatorname{Ji}(L), x \leq b\}$ join-generates $[a, b]$.

Let $\operatorname{Diag}(L)$ stand the set of all planar diagrams of $L$. The general convention throughout the paper is that a planar diagram $D \in \operatorname{Diag}(L)$ is fixed, unless otherwise stated. Many concepts we are going to define depends on the choice of $D$, at least seemingly. However, in several cases we will prove that this dependence is only apparent without being real. The diagram $D$ divides the plane into minimal 
regions, which are called cells. In presence of semimodularity, all cells are covering squares (i.e., cover-preserving four-element Boolean sublattices), so these cells are called 4-cells. While 4-cells are always covering squares, (the usual diagram of) $M_{3}$ indicates that the converse fails even for modular planar lattices in general; indeed, $M_{3}$ has three covering squares but only two 4-cells. By [7, Prop. 1], for any planar semimodular lattice $L$ and for an arbitrary $D \in \operatorname{Diag}(L)$,

$L$ is slim iff all of its covering squares are 4-cells.

This is the original definition of slimness in [15] for the semimodular case. Notice that the expression "4-cells" in (2.3) is a short form of the more precise "4-cells of the fixed diagram $D$ " or "its 4-cells with respect to $D$ "; similar terminology will frequently occur. A lattice $L$ (in particular, a chain) is called nontrivial if it contains at least two elements. If $L$ is a non-chain lattice and for each $x \in L \backslash\{0,1\}$, there is a $y \in L$ such that $x$ and $y$ are incomparable, then $L$ is called glued sum indecomposable.

Given a fixed planar diagram $D$ of a lattice $L$, it has a left boundary chain $\mathcal{B}_{\text {left }}(L)=\mathcal{B}_{\text {left }}^{D}(L)$, a right boundary chain $\mathcal{B}_{\text {right }}(L)=\mathcal{B}_{\text {right }}^{D}(L)$, and a boundary $\mathcal{B}(L)=\mathcal{B}^{D}(L)=\mathcal{B}_{\text {left }}(L) \cup \mathcal{B}_{\text {right }}(L)$. (Here and in similar situations, if there is no danger of confusion, we often drop $D$ from the notation.) The interior of $L$, denoted by $\operatorname{int}^{D}(L)$ or simply by $\operatorname{int}(L)$, is $L \backslash \mathcal{B}^{D}(L)$. For a rigorous treatment of these concepts see Kelly and Rival [23]. Notice that, by [23, Prop. 2.2],

$$
\operatorname{Ji}(L) \cap \operatorname{Mi}(L) \cap \mathcal{B}_{\text {left }}(L) \neq \emptyset \quad \text { and } \quad \operatorname{Ji}(L) \cap \operatorname{Mi}(L) \cap \mathcal{B}_{\text {right }}(L) \neq \emptyset,
$$

provided $|L| \geq 3$. We have to recall some further concepts and properties of planar lattices from [23]. Let $L$ be a planar lattice with a fixed planar diagram $D$. If $C$ is a maximal chain of $L$, then it has a left side, denoted by $\operatorname{left}_{\text {side }}^{D}(C)$, and a right side right $_{\text {side }}^{D}(C)$. Notice that $\operatorname{left}_{\text {side }}^{D}(C) \cup \operatorname{right}_{\text {side }}^{D}(C)=L$ and $C=\operatorname{left}_{\text {side }}^{D}(C) \cap \operatorname{right}_{\text {side }}^{D}(C)$. The strict sides of $C$ (with respect to $D$ ) are $\operatorname{left}_{\text {side }}^{D}(C) \backslash C$ and $\operatorname{right}_{\text {side }}^{D}(C) \backslash C$. If $a \leq b$ in $L,|[a, b]| \geq 3$ and $C_{1}$ and $C_{2}$ are maximal chains of $[a, b]$ such that $C_{1} \cap C_{2}=\{a, b\}$, then $C_{1}$ and $C_{2}$ determines a socalled region $R$ of $L$. It is a convex sublattice with $\left\{\mathcal{B}_{\text {left }}(R), \mathcal{B}_{\text {right }}(R)\right\}=\left\{C_{1}, C_{2}\right\}$. Its interior, $\operatorname{int}(R)$ is $R \backslash \mathcal{B}(R)$. Assume that $u \in R$ and $v \in L \backslash R$, or conversely. Let $a, b, c \in L$. Further, let $C$ be a maximal chain of $L$, and let $x, y \in L$ such that $x$ and $y$ are on different sides of $C$. Then, by [23, Lemmas 1.2 and 1.5], by the definition of a region, and by (2.7),

$$
\begin{aligned}
& \text { If } x \leq y \text {, then there is a } z \in C \text { with } x \leq z \leq y \text {; } \\
& \text { every interval is a region; } \\
& \text { if } u \leq v \text {, then there is a } w \in \mathcal{B}(R) \text { with } u \leq w \leq v \text {; } \\
& \text { if } b \in \operatorname{int}(R) \text { and } a \prec b \prec c \text {, then } a, c \in R \text {. }
\end{aligned}
$$

When referring to properties of regions, we often use (2.6) implicitly. By the exact definition of a region, given in [23], we also have that

$$
\text { if } R \text { is a region, then } \operatorname{int}(R) \subseteq \operatorname{int}(L) .
$$

For slim lattices, we can assert even more. By [7, Lemma 6],

$$
\mathrm{Ji}(L) \subseteq \mathcal{B}^{D}(L), \text { provided } L \text { is slim. }
$$

Also, if $L$ is slim, then $\mathcal{B}(L)$ is uniquely determined by [7, Lemma 7]. That is, $\mathcal{B}^{D}(L)=\mathcal{B}^{F}(L)$, for all $D, F \in \operatorname{Diag}(L)$. By [7, Lemma 7], if $L$ is slim and glued 
sum indecomposable, then even $\left\{\mathcal{B}_{\text {left }}(L), \mathcal{B}_{\text {right }}(L)\right\}$ is uniquely determined. That is, the left and th right boundary chains are determined up to symmetry. (For a stronger statement, see Lemma 4.7 later.)

Let $L$ be a planar semimodular lattice with a fixed $D \in \operatorname{Diag}(L)$. By a weak corner of $D$ we mean a doubly irreducible element $d$ on the boundary of $L$ such that $d$ is distinct from 0 and 1. (Sometimes we speak of weak corners of $L$ even if they depend on D.) Following Grätzer and Knapp [16], by a rectangular lattice we mean a planar semimodular lattice $L$ such that $L$ has a planar diagram $D$ such that $\mathcal{B}_{\text {left }}^{D}(L)$ has exactly one weak corner, denoted by $w_{L}^{\ell}, w_{D}^{\ell}$ or $w_{D}^{\ell}(L), \mathcal{B}_{\text {right }}^{D}(L)$ has exactly one weak corner, denoted by $w_{L}^{r}, w_{D}^{r}$ or $w_{D}^{r}(L)$, and they are complementary, that is, $w_{D}^{\ell} \wedge w_{D}^{r}=0$ and $w_{D}^{\ell} \vee w_{D}^{r}=1$. (Although the weak corners depend on $D$, Lemma 5.5 will show later that all planar diagrams are equally appropriate to check whether $L$ is rectangular.) Clearly, rectangular lattices have at least four elements and they cannot be chains. If $L$ is slim, then, by the already mentioned [7, Lemma $7],\left\{\mathcal{B}_{\text {left }}^{D}(L), \mathcal{B}_{\text {right }}^{D}(L)\right\}$ and $\left\{w_{D}^{\ell}(L), w_{D}^{r}(L)\right\}$ do not depend on the planar diagram chosen. (In fact, Lemma 4.7 will state even more.) It is easy to see (and we know it from [7, before Prop. 10]) that for a slim (not just planar) semimodular lattice $L, L$ is rectangular iff $\operatorname{Ji}(L)$ is the union of two disjoint chains $C$ and $W$ such that every element of $C$ is incomparable with all elements of $W$.

For a rectangular lattice $L$ and $D \in \operatorname{Diag}(L)$, we define the top boundary chains $\mathcal{B}_{\mathrm{nw}}^{D}(L)=\uparrow w_{D}^{\ell} \cap \mathcal{B}_{\text {left }}^{D}(L)$ and $\mathcal{B}_{\text {ne }}^{D}(L)=\uparrow w_{D}^{r} \cap \mathcal{B}_{\text {right }}^{D}(L)$, the bottom boundary chains $\mathcal{B}_{\text {sw }}^{D}(L)=\downarrow w_{D}^{\ell} \cap \mathcal{B}_{\text {left }}^{D}(L)$ and $\mathcal{B}_{\text {se }}^{D}(L)=\downarrow w_{D}^{r} \cap \mathcal{B}_{\text {right }}^{D}(L)$, the northern boundary $\mathcal{B}_{\text {north }}^{D}(L)=\mathcal{B}_{\text {nw }}^{D}(L) \cup \mathcal{B}_{\text {ne }}^{D}(L)$, and the southern boundary $\mathcal{B}_{\text {south }}^{D}(L)=\mathcal{B}_{\mathrm{sw}}^{D}(L) \cup \mathcal{B}_{\mathrm{se}}^{D}(L)$. We know from Grätzer and Knapp [16, Lemmas 3 and 4] and from the definition of a rectangular lattice that, for each rectangular lattice $L$,

$$
\begin{gathered}
\mathcal{B}_{\mathrm{nw}}^{D}(L), \mathcal{B}_{\mathrm{ne}}^{D}(L), \mathcal{B}_{\mathrm{sw}}^{D}(L) \text { and } \mathcal{B}_{\mathrm{se}}^{D}(L) \text { are indeed chains, } \\
\mathcal{B}_{\mathrm{nw}}^{D}(L)=\uparrow w_{D}^{\ell}, \quad \mathcal{B}_{\mathrm{ne}}^{D}(L)=\uparrow w_{D}^{r}, \quad \mathcal{B}_{\mathrm{sw}}^{D}(L)=\downarrow w_{D}^{\ell}, \quad \mathcal{B}_{\mathrm{se}}^{D}(L)=\downarrow w_{D}^{r}, \\
\mathcal{B}_{\text {north }}^{D}(L) \backslash\{1\} \subseteq \operatorname{Mi}(L), \quad \mathcal{B}_{\text {south }}^{D}(L) \backslash\{0\} \subseteq \mathrm{Ji}(L), \\
\text { each element of } \mathcal{B}^{D}(L) \backslash \mathcal{B}_{\text {north }}^{D}(L) \text { has at least two covers, and } \\
\text { each element of } \mathcal{B}^{D}(L) \backslash \mathcal{B}_{\text {south }}^{D}(L) \text { has at least two lower covers. }
\end{gathered}
$$

Let $d$ be a doubly irreducible element of a slim semimodular lattice $L$. Then $d$ belongs to (at least) one of the boundary chains $\mathcal{B}_{\text {left }}^{D}(I)$ and $\mathcal{B}_{\text {right }}^{D}(I)$ by $(2.10)$. Since this boundary chain is a maximal chain, it contains the unique lower cover $d^{-}$and the unique upper cover $d^{+}$of $d$. If $d^{-}$has exactly two upper covers and $d^{+}$has exactly two lower covers, then $d$ is called a corner of $D$. Note that corners are weak corners but (even for rectangular lattices) not conversely. A corner can be removed and a slim semimodular sublattice remains by [7, Prop. 10].

Some earlier structure theorems. Let $S$ be a 4-cell of a planar diagram $D$ of a planar lattice $L$. Replace this 4-cell by a copy of $M_{3}$, the five-element nondistributive modular lattice (with a fixed diagram). This means that we insert a new element, which is called an eye, into the interior of $S$, and this way we divide $S$ into two new 4-cells. This way we obtain a new diagram that determines a new lattice. If $D^{\bullet}$ and $L^{\bullet}$ is obtained from $L$ by inserting eyes one by one, then $D^{\bullet}$ and $L^{\bullet}$ is called an anti-slimming of $D$ and $L$, respectively, and $D^{\bullet} \in \operatorname{Diag}\left(L^{\bullet}\right)$. A 

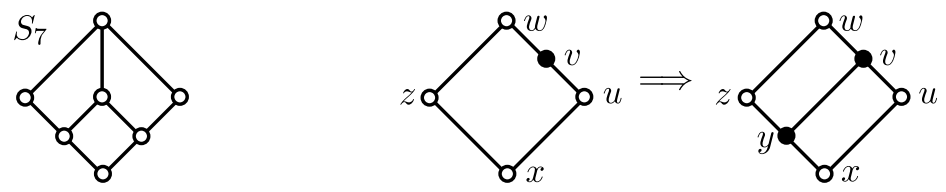

FiguRE $1 . S_{7}$ and the downward-going procedure

0-1-sublattice means a sublattice with the same 0 and 1 . We recall the following statement.

Proposition 2.1 (Grätzer and Knapp [15]). Each planar semimodular lattice L is an anti-slimming of one of its slim semimodular 0-1-sublattices, $L^{\prime}$.

Proposition 2.1 reduces most of the questions on planar semimodular lattices to the slim case. Let $D \in \operatorname{Diag}(L)$ be fixed. Then the sublattice $L^{\prime}$ (with the corresponding diagram $D^{\prime}$ ) above is called a full slimming sublattice of $L$. More exactly, $D^{\prime}$ is obtained from $D$ by omitting all elements from the interiors of intervals of length two. For a fixed $L^{\prime}$ (which depends only on $D$ ), the elements of $L \backslash L^{\prime}$ (or those of $D \backslash D^{\prime}$ ) are called eyes. Clearly, for each eye $e \in L \backslash L^{\prime}$, if $e^{-}$and $e^{+}$ denote the unique lower and upper cover of $e$, respectively, then

$$
e^{-} \text {and } e^{+} \text {belong to a unique 4-cell }\left\{e^{-}, a, b, e^{+}\right\} \text {of } L^{\prime} \text {. }
$$

Let us emphasize the difference between a full slimming sublattice of $L$, which is a sublattice (a concrete subset of $L$ ) and depends on $D$, and the full slimming of $L$, which is an abstract lattice, not a concrete sublattice of $L$. While $L$ can have many full slimming sublattices, as witnessed by $L=M_{3}$, the full slimming of $L$ will turn out to be unique, see Remark 4.2.

The first structure theorem for slim semimodular lattices is due to Grätzer and Knapp [15], and it was soon generalized in [4]. (We have recently discovered that even the generalized version was already present but well-hidden in Stern [25]. However, it is [15] that initiated a rapid development leading to the present work.) Other structure theorems were given in [7] (two theorems), [2], and [8]; we will need and recall only one of them. Let $S$ be a 4-cell of a slim semimodular lattice $L$, with respect to $D \in \operatorname{Diag}(L)$. Then $S$ is a covering square $\left\{a=b_{1} \wedge b_{2}, b_{1}, b_{2}\right.$, $\left.c=b_{1} \vee b_{2}\right\}$. We change $L$ to a new lattice $L^{*}$ as follows. Firstly, we replace $S$ by a copy of $S_{7}$; see Figure 1 for its definition. This way we get three new 4-cells instead of $S$. Secondly, as long as there is a chain $u \prec v \prec w$ such that $v$ is a new element and $T=\{x=u \wedge z, z, u, w=u \vee z\}$ is a 4-cell in the original lattice $L$ but $x \prec z$ at the present stage, see Figure 1, we insert a new element $y$ such that $x \prec y \prec z$ and $y \prec v$. (This way we get two 4-cells to replace the 4-cell T.) When this "downward-going" procedure terminates, we obtain $L^{*}$. The collection of all new elements, which is an order (also called poset), will be called a fork. We say that $L^{*}$ is obtained from $L$ by adding a fork to $L$ (at the 4 -cell $S$ ). For an illustration, see see Figure 2, where $L_{i}$ is obtained from $L_{i-1}$ by adding a single fork; the new elements of $L_{i}$, which form a fork, are the black-filled ones. Adding forks to $L$ means adding several forks to $L$ one by one. For example, $L_{3}$ in Figure 2 is obtained from $L_{0}=\mathbf{2}^{2}$ by adding forks, in three steps. By a grid we mean the direct product of two finite, nontrivial chains. (The smallest grid is $\mathbf{2}^{2}$.) We are now ready to recall 

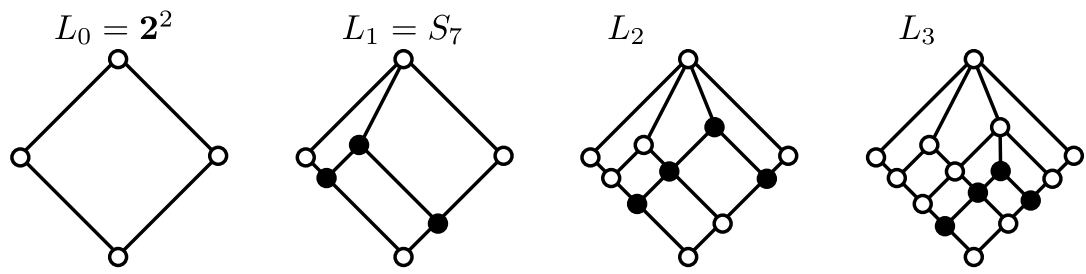

FIGURE 2. Forks and slim patch lattices

Proposition 2.2 ([7, Theorem 12]). Let L be a slim semimodular lattice consisting of at least three elements. Then $L$ can be obtained from a grid such that

(i) first we add finitely many (possibly zero) forks one by one,

(ii) and then we remove some (possibly zero) corners, one by one.

For later reference, we formulate a trivial statement, see also [9, Figure 1].

Lemma 2.3. Each nontrivial finite lattice is uniquely decomposable as a glued sum of nontrivial chains and glued sum indecomposable lattices.

Rectangular lattices are of separate interest, not only in the present paper but also in Grätzer and Knapp [16], [17], [3] and [24]. In connection with parts (iv) and (vii) of (the forthcoming) Theorem 3.4, we present the following structure theorem for them. Remember that grids are defined right before Proposition 2.2.

Proposition 2.4 (Mainly [7, Lemma 22], as detailed in Section 10). Let $L$ be an arbitrary slim rectangular lattice. Then

(i) there is a grid $G$ such that $L$ can be obtained from $G$ by adding forks;

(ii) For all $D \in \operatorname{Diag}(L), G$ is (isomorphic to) $\uparrow w_{D}^{\ell}(L) \times \uparrow w_{D}^{r}(L)$. Consequently, $G$ is uniquely determined up to isomorphism.

(iii) Every lattice obtained from a grid by adding forks is a slim rectangular lattice.

(iv) Each rectangular lattice is an anti-slimming of a slim rectangular lattice, which is unique up to isomorphism.

\section{PATCHWORK SYSTEMS AND THE NEW RESULTS}

An interval is called a rectangular interval, if it is a rectangular lattice. As usual, $\mathbb{N}$ and $\mathbb{N}_{0}$ stand for the set of positive integers and $\mathbb{N} \cup\{0\}$, respectively. We will deal only with glued sum indecomposable lattices. In virtue of Lemma 2.3, this reasonable restriction is not a serious loss since finite chains and the glued sum construction are well-understood.

Definition 3.1. Let $L$ be a glued sum indecomposable planar semimodular lattice, and let $\mathcal{H}$ be a collection of rectangular intervals of $L$. Let $\mathcal{E}(\boldsymbol{H})$ denote the set $\left\{(I, J) \in \mathcal{H}^{2}: I \neq J\right.$ and $\left.I \cap J \neq \emptyset\right\}$. We say that $\mathcal{H}$ is a patchwork system for $L$, if the following three conditions hold:

(i) For each covering square $S$ of $L$, there exists an $I \in \mathcal{H}$ such that $S \subseteq I$.

(ii) For all $(I, J) \in \mathcal{E}(\mathcal{H}), I \cap J$ is a chain.

(iii) $L$ has a planar diagram $D$ such that, for all $(I, J) \in \mathcal{E}(\mathcal{H})$, we have that $I \cap J \subseteq \mathcal{B}_{\text {north }}^{D}(I) \cap \mathcal{B}_{\text {south }}^{D}(J)$ or $I \cap J \subseteq \mathcal{B}_{\text {south }}^{D}(I) \cap \mathcal{B}_{\text {north }}^{D}(J)$. 
If $\mathcal{H}$ is a patchwork system of $L$ such that $D \in \operatorname{Diag}(L)$ witnesses (iii), then we also say that $\mathcal{H}$ is a patchwork system for the diagram $D$. Hence $\mathcal{H}$ is a patchwork system for $L$ iff it is a patchwork system for some $D \in \operatorname{Diag}(L)$. A patchwork system $\mathcal{H}$ is nontrivial if $|\mathcal{H}| \geq 2$.

Sometimes we say that $L$ is sewn from the members of $\mathcal{H}$. If there is a patchwork system for $L$, then we also say that $L$ allows a patchwork system. An example of a patchwork system for $L$ is provided by Figure 3 ; this system consists of eleven rectangular intervals: four light grey ones, five dark gray ones and two striped ones. The following statement sheds more light on this concept, and it offers the possibility of several equivalent definitions. Condition (i) of Definition 3.1 will be referenced as 3.1(i), and a similar convention will apply for conditions occurring in statements.

Proposition 3.2. Assume that $\mathcal{H}$ is a set of rectangular intervals of a glued sum indecomposable planar semimodular lattice $L$ such that $\mathcal{H}$ satisfies $3.1(\mathrm{i})$ and 3.1(ii). Then the following four conditions are equivalent.

(i) $\mathcal{H}$ satisfies 3.1 (iii), that is, $\mathcal{H}$ is a patchwork system for $L$.

(ii) For all planar diagrams $D$ of $L$ and for for all $(I, J) \in \mathcal{E}(\mathcal{H})$, we have that $I \cap J \subseteq \mathcal{B}_{\text {north }}^{D}(I) \cap \mathcal{B}_{\text {south }}^{D}(J)$ or $I \cap J \subseteq \mathcal{B}_{\text {south }}^{D}(I) \cap \mathcal{B}_{\text {north }}^{D}(J)$. That is, $\mathcal{H}$ is a patchwork system for all $D \in \operatorname{Diag}(L)$.

(iii) There exists a planar diagram $D$ of $L$ such that for each $(I, J) \in \mathcal{E}(\mathcal{H})$,

(a) $I \cap J \subseteq \mathcal{B}_{\text {north }}^{D}(I) \cap \mathcal{B}_{\text {south }}^{D}(J)$ or $I \cap J \subseteq \mathcal{B}_{\text {south }}^{D}(I) \cap \mathcal{B}_{\text {north }}^{D}(J)$, and

(b) $I \cap J \subseteq \mathcal{B}_{\text {left }}^{D}(I) \cap \mathcal{B}_{\text {right }}^{D}(J)$ or $I \cap J \subseteq \mathcal{B}_{\text {right }}^{D}(I) \cap \mathcal{B}_{\text {left }}^{D}(J)$.

(iv) The previous two subconditions, 3.2(iiia) and 3.2(iiib), hold for all planar diagrams $D$ of $L$ and for each $(I, J) \in \mathcal{E}(\mathcal{H})$.

Remark 3.3. Let $L$ be and $\mathcal{H}$ be as in Proposition (3.2).

(i) If $I$ and $J$ are distinct members of a patchwork system $\mathcal{H}$, then $I$ and $J$ are incomparable (in notation, $I \| J$ ), that is, $I \nsubseteq J$ and $J \nsubseteq I$. (This follows from 3.1(ii) since a rectangular interval is never a chain.)

(ii) Since $\mathcal{B}_{\text {left }}(I)$ and $\mathcal{B}_{\text {right }}(I)$ are always chains, 3.2(iiib) implies 3.1(ii).

(iii) It will follow from 3.1(i) and Lemma 4.3 that $\mathcal{H}$ covers $L$ in the sense that $L=\bigcup_{I \in \mathcal{H}} I$.

(iv) Let $\mathcal{G}$ be a set of rectangular intervals of $L$. Then 3.1(i) holds for $\mathcal{G}$ iff $L$ has a planar diagram $D$ such that each 4-cell of $D$ is a subset of some member of $\mathcal{G}$ iff for every a planar diagram $D$ of $L$ each 4-cell of $D$ is a subset of some member of $\mathcal{G}$.

(v) The purpose of $3.1(\mathrm{i})$ is to ensure something like " $\mathcal{H}$ is simply connected" (in other words, 1-connected) in topological sense. For example, if $L=\mathbf{3}^{2}$ and $\mathcal{G}$ is the collection of all covering squares, then $\mathcal{G}$ is a patchwork system for $L$. However, if the middle square $S$ is removed, then $\mathcal{G} \backslash\{S\}$ is not a patchwork system since 3.1(i) fails (while 3.1(ii) and 3.1(iii) hold).

We call a slim semimodular lattice $L$ patchwork-irreducible, if it allows a patchwork system and, in addition, for every patchwork system $\mathcal{H}$ for $L,|\boldsymbol{H}|=1$. In other words, if $L$ is rectangular and it allows only the trivial patchwork system. For example, $S_{7}$ in Figure 1 is patchwork-irreducible. To define two related but more classical concepts, let $L$ be a nontrivial lattice. If there are a proper ideal $I$ and a proper filter $F$ such that $I \cap F$ is nonempty and $L=I \cup F$, then $L$ is decomposable 


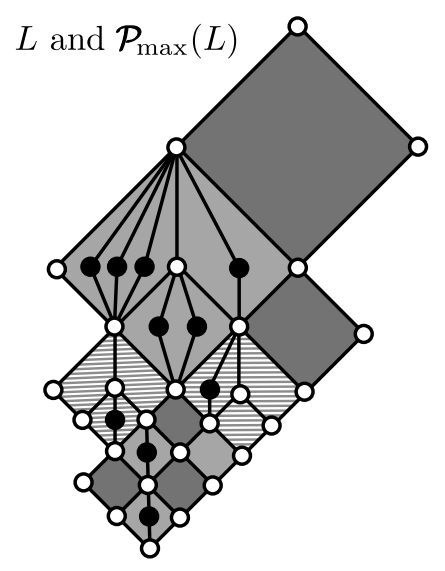

Figure 3. A patchwork system

with respect to the Hall-Dilworth gluing (in the general sense), HDg-decomposable for short. If $L$ is not a chain (equivalently, $|L| \geq 3$ or, still equivalently, $|L| \geq 4$ ) and $L$ is not HDg-decomposable, then we say that $L$ is indecomposable with respect to the Hall-Dilworth gluing, HDg-indecomposable for short. Notice that the two-element lattice is neither HDg-decomposable, nor HDg-indecomposable.

Similarly, assume that $L$ is not a chain (equivalently, $|L| \geq 3$ or, still equivalently, $|L| \geq 4$ ), and whenever $I$ is an ideal and $F$ is a filter of $L$ such that $I \cap F$ is a chain and $L=I \cup F$, then $L \in\{I, F\}$. Then we say that $L$ is indecomposable with respect to the Hall-Dilworth gluing over chains, HDc-indecomposable for short. Each of patchwork-irreducibility, HDg-indecomposability and HDc-indecomposability implies that our lattice is not a chain, it is glued sum indecomposable and consists of at least four elements.

Theorem 3.4. Let $L$ be a planar semimodular lattice. Assume that $|L| \geq 4$. Then the following seven conditions are equivalent.

(i) $L$ is a patchwork-irreducible lattice;

(ii) $L$ is indecomposable with respect to the Hall-Dilworth gluing;

(iii) $L$ is indecomposable with respect to the Hall-Dilworth gluing over chains;

(iv) $L$ is a rectangular lattice whose weak corners $w_{D}^{\ell}$ and $w_{D}^{r}$, with respect to some planar diagram $D$ of $L$, are coatoms;

(v) L has a planar diagram such that the intersection of the leftmost coatom and the rightmost coatom is 0 ;

(vi) for each planar diagrams of $L$, the intersection of the leftmost coatom and the rightmost coatom is 0 ;

(vii) $L$ is an anti-slimming of a lattice obtained from the four-element Boolean lattice by adding finitely many forks one by one.

By a patch lattice we mean a rectangular lattice $L$ whose weak corners, with respect to some $D \in \operatorname{Diag}(L)$, are coatoms; that is, a lattice satisfying 3.4(iv) above. Theorem 3.4 offers six alternative definitions. Some slim patch lattices are given in Figure 2. Some non-slim patch lattices occur among the members of $\mathcal{P}_{\max }(L)$ in Figure 3. Theorem 3.4 trivially leads to the following structure theorem, 
which, opposed to Proposition 2.2 (and the other theorem of [7]), does not need the concept of a corner.

Corollary 3.5 (A structure theorem). Each planar semimodular lattice can be constructed as the last member of a finite list $L_{1}, L_{2}, \ldots, L_{n}$ such that each $L_{i}$ $(i=1, \ldots, n)$ is either a patch lattice (constructed according to Theorem 3.4(vii)), or there are $j, k<i$ such that $L_{i}$ is a Hall-Dilworth gluing of $L_{j}$ and $L_{k}$ over a chain. Conversely, every lattice constructed this way is a planar semimodular lattice.

A patch of a lattice is an interval that is a patch lattice. Let $\mathcal{P}(L)$ denote the set of all patches of $L$, and let $\boldsymbol{P}_{\max }(L)$ be the set of maximal patches of $L$ (with respect to set inclusion). Our third structure theorem is the following one.

Theorem 3.6. Let $L$ be a glued sum indecomposable planar semimodular lattice. Then $\mathcal{P}_{\max }(L)$ is a patchwork system for $L$.

For example, if $L$ is the (glued sum indecomposable planar semimodular) lattice given by Figure 3, then $\boldsymbol{P}_{\max }(L)$ is depicted in the same figure. This $L$ is not slim. If we deleted all the black-filled elements, then we would obtain a slim lattice $L^{\prime}$, and the figure would depict $\boldsymbol{P}_{\max }\left(L^{\prime}\right)$.

Since $S_{7}$ is not a modular lattice, in the modular case we cannot add forks. Similarly, in the distributive case we cannot add eyes. Hence Theorems 3.4 and 3.6 together with Proposition 2.1 clearly imply the following two corollaries (except for the last sentence of the second one). The first of them is a folklore result (with another terminology), see also Grätzer and Knapp [15, Introduction].

Corollary 3.7. If $L$ is a glued sum indecomposable planar distributive lattice, then $\mathcal{P}_{\max }(L)$ is the set of all 4 -cells, and it is a patchwork system for $L$.

The definition of Herrmann's $S$-glued systems will not be needed here; the reader can see [20] for details. The main result of Herrmann [20] asserts that the maximal complemented (equivalently, maximal atomistic) intervals of a modular lattice $M$ of finite length form an $S$-glued system, which we denote by $\mathcal{H}^{\mathrm{Herrm}}(M)$.

Corollary 3.8. If $L$ is a glued sum indecomposable planar modular lattice, then $\mathcal{P}_{\max }(L)$ is the set of all non-chain intervals of length 2. Moreover, the patchwork system $\boldsymbol{P}_{\max }(L)$ coincides with the $S$-glued system $\boldsymbol{\mathcal { H }}^{\mathrm{Herrm}}(L)$.

Hence Theorem 3.6 extends the main result of Herrmann [20] to planar semimodular lattices. However, there is an essential difference. If $M$ is a modular lattice, then $\varrho:=\bigcup\left\{A^{2}: A \in \mathcal{H}^{\operatorname{Herrm}}(M)\right\}$ is a lattice tolerance, see Day and Herrmann [10], and the quotient lattice $L / \rho$ in the sense of [1] is what Herrmann calls the "skeleton" of his construction. However, if $L$ is (the planar semimodular) lattice given in Figure 3, then $\varrho:=\bigcup\left\{A^{2}: A \in \mathcal{P}_{\max }(L)\right\}$ is not a lattice tolerance. Hence we do not associate "skeleton lattices" with patchwork systems.

\section{More about planar Diagrams}

Lemma 4.1. Let $L_{i}^{\prime}$ be a full slimming sublattice of a planar semimodular lattice $L_{i}$, for $i \in\{1,2\}$. If $L_{1}$ is isomorphic to $L_{2}$, then $L_{1}^{\prime}$ is isomorphic to $L_{2}^{\prime}$.

Remark 4.2. This lemma allows us to speak of the full slimming of a slim semimodular lattice $L$ : it is any of the full slimming sublattices of $L$, and it is considered 
an abstract lattice. Lemma 4.1 implies that the full slimming $L^{\prime}$ of $L$ is uniquely determined up to isomorphism. In other words, the isomorphism type of $L^{\prime}$ does not depend on the planar diagram of $L$.

Proof of Lemma 4.1. We apply induction by $\left|L_{1}\right|$. Let $D_{i}$ be a planar diagram of $L_{i}$, for $i \in\{1,2\}$. Let $\varphi: L_{1} \rightarrow L_{2}$ be an isomorphism. If $L_{1}$ is slim, then the statement is trivial. Assume that $L_{1}$ is not slim. Then there are $u<v \in L_{1}$ such that $[u, v]$ is an interval of length two and $[u, v]$ contains a doubly irreducible element $s_{1}$ that belongs to $\operatorname{int}^{D_{1}}([u, v])$ (the interior of $[u, v]$ with respect to the diagram $D_{1}$ ). Let $s_{2}$ be a doubly irreducible element of $L_{2}$ that belongs to $\operatorname{int}^{D_{2}}([\varphi(u), \varphi(v)])$. Then $t:=\varphi^{-1}\left(s_{2}\right)$ is a doubly irreducible element in $L_{1}$, and it belongs to $[u, v]$. Obviously, there is an automorphism of $L_{1}$ that sends $s_{1}$ to $t$ and $t$ to $s_{1}$, and keeps any other element fixed. Let $\psi$ denote the composite of this automorphism and $\varphi$. Then $\psi: L_{1} \rightarrow L_{2}$ is an isomorphism and $\psi\left(s_{1}\right)=s_{2}$.

Let $L_{i}^{-}:=L_{i} \backslash\left\{s_{i}\right\}$, for $i \in\{1,2\}$, and let $D_{i}^{-}$denote the diagram obtained from $D_{i}$ by removing $s_{i}$. The restriction $\psi^{-}$of $\psi$ to $L_{1}^{-}$is an isomorphism $\psi^{-}: L_{1}^{-} \rightarrow L_{2}^{-}$. Clearly, $L_{i}^{\prime}$ is the full slimming sublattice of $L_{i}^{-}$with respect to $D_{i}^{-}$, for $i=1,2$. Since $\left|L_{1}^{-}\right|<L_{1}$, the induction hypothesis applies, and we obtain that $L_{1}^{\prime} \cong L_{2}^{\prime}$.

Two-element intervals are called prime intervals. That is, $[a, b]$ is a prime interval iff $a \prec b$. A covering square $B$ is formed by four edges, which are the prime intervals of $B$. Opposed to 4-cells of a given diagram, covering squares need not be regions.

Lemma 4.3. Let $[a, b]$ be a prime interval of a glued sum indecomposable planar semimodular lattice. Then a is meet-reducible or $b$ is join-reducible. Furthermore, $[a, b]$ is an edge of a covering square. Moreover, for any fixed planar diagram of $L$, $[a, b]$ is an edge of a 4 -cell of $D$.

Proof. By way of contradiction, assume that $a \prec b$ such that $a \in \operatorname{Mi}(L)$ and $b \in \operatorname{Ji}(L)$. Since $L$ is glued sum indecomposable, we can select a minimal $y \in L$ such that $y \| b$. Then $y \neq 0$, so it has a lower cover $x$. By the minimality of $y$, we have that $x<b$, which gives that $x \leq a$. Semimodularity yields that $a=a \vee x \preceq a \vee y$. This means that $a \vee y$ is $a$ or $b$ since $b$ is the only cover of $a$. However, both possibilities lead to $y \leq b$, a contradiction. Thus, $a \notin \operatorname{Mi}(L)$ or $b \notin \mathrm{Ji}(L)$, proving the first part of the lemma.

If $a$ is meet-reducible, then it has a cover $c$ distinct from $b$, and $S=\{a, b, c, b \vee c\}$ is a covering square by semimodularity. The prime interval $[a, b]$ is an edge of $S$. If we chose $c$ such that $b$ and $c$ are neighboring covers of $a$ (in the fixed diagram $D$ ), then $S$ is a 4-cell. Next, assume that $b$ is join-reducible. Then, with respect to $D$, there is a $c \in L$ such that $a$ and $c$ are neighboring lower covers of $b$. Then $[a \wedge c, b]$ is a 4 -cell by [7, Lemma 13], and $[a, b]$ is one of its edges.

On the set $\operatorname{Prin}(L)$ of all prime intervals of $L$, we define a relation $\mu$ as follows: for $\mathfrak{p}, \mathfrak{q} \in \operatorname{Prin}(L)$, let $\mathfrak{p} \mu \mathfrak{q}$ mean that there is a covering square $B$ such that both $\mathfrak{p}$ and $\mathfrak{q}$ are edges of $B$. We will also need a similar relation defined on $\operatorname{Prin}(D)=\operatorname{Prin}(L)$, where $D \in \operatorname{Diag}(L)$. For $\mathfrak{p}, \mathfrak{q} \in \operatorname{Prin}(L)$, let $\mathfrak{p} \varrho_{D} \mathfrak{q}$ mean that there is a 4 -cell $B$ in the diagram $D$ such that both $\mathfrak{p}$ and $\mathfrak{q}$ are edges of $B$. Both $\mu$ and $\varrho_{D}$ are reflexive and symmetric relations, provided $L$ belongs to the scope of Lemma 4.3. Their transitive closures will be denoted by $\mu^{*}$ and $\varrho_{D}^{*}$. 
Lemma 4.4. Let $L$ be a glued sum indecomposable planar semimodular lattice, and let $D \in \operatorname{Diag}(L)$. Then $\mu^{*}$ and $\varrho_{D}^{*}$ are the "full relation" $\operatorname{Prin}(L) \times \operatorname{Prin}(L)$ on the set of prime intervals of $L$.

Proof. Clearly, $\mu \subseteq \varrho_{D}^{*}$. Therefore, it suffices to deal with $\mu$. Let $[u, v] \in \operatorname{Prin}(L)$. By induction on the height $h(v)$ of $v$, we are going to show that

$$
\text { there is an atom } r \in L \text { such that }[0, r] \mu^{*}[u, v] \text {. }
$$

By reflexivity, this is trivial for $h(v)=1$. So let $h(v) \geq 2$. Let $a$ and $b$ be the leftmost lower cover and the rightmost lower cover of $u$, respectively. (They are not distinct in general, and they are never distinct if $h(v)=2$.) Let $H$ be a maximal chain in $\uparrow v$. Then $W:=\mathcal{B}_{\text {left }}^{D}(\downarrow u) \cup H$ and $E:=\mathcal{B}_{\text {right }}^{D}(\downarrow u) \cup H$ are maximal chains of $L$, and $a \in W$ and $b \in E$. These two maximal chains divide $L$ into the strict left side $L_{W}:=\operatorname{left}_{\text {side }}^{D}(W) \backslash W$ of $W$, the strict right side $R_{E}:=\operatorname{right}_{\text {side }}^{D}(E) \backslash E$ of $E$, and $H \cup \downarrow u=\operatorname{right}_{\text {side }}^{D}(W) \cap \operatorname{left}_{\text {side }}^{D}(E)$. Since $L$ is glued sum indecomposable, there is an $x \in L$ such that $x \| u$. We assume that $x$ is minimal with respect to this property. By left-right symmetry, we can also assume that $x \in R_{E}$. There are two cases.

Assume first that $b \leq x$. Then $b<x$ since $x \| u$ and $b<u$. Take an atom $x^{\prime}$ in the interval $[b, x]$. Then $u \not z x$ gives that $x^{\prime} \neq u$. Hence, as two covers of $b, x^{\prime}$ and $u$ are incomparable. Since $x$ was minimal with respect to this property, we obtain that $x^{\prime}=x$. That is, we have the situation

there is an $x \in R_{E}$ such that $x \neq u$ and $b \prec x$.

Let $t=u \vee x$. Then $\{b, u, x, t\}$ is a covering square by semimodularity. If $t \neq v$, then $\{u, v, t, v \vee t\}$ is another covering square. Since $h(u)<h(v)$, the induction hypothesis yields an atom $r \in L$ such that $[0, r] \mu^{*}[b, u]$. The covering square $\{b, u, x, t\}$ gives that $[b, u] \mu^{*} s q[u, t]$. Hence $[u, t] \mu^{*}[u, v]$ follows either from $v=t$ and reflexivity, see Lemma 4.3 , or from the covering square $\{u, v, t, v \vee t\}$. By transitivity, $[0, r] \mu^{*}[u, v]$, as desired.

Secondly, we assume that $b \not \leq x$, that is, $b<b \vee x$. Since $x \| u, x$ has a lower cover $x_{0}$. The minimality of $x$ gives that $x_{0}<u$. Hence $x_{0}$ is on the left side of $E$ while $x \in R_{E}$ is on the strict right side of $E$. We conclude from (2.5) and $x_{0} \prec x$ that $x_{0} \in E$. Hence $x_{0} \in \mathcal{B}_{\text {right }}(\downarrow u)$. Since $\mathcal{B}_{\text {right }}(\downarrow u)$ is a chain and $x \neq u$, we obtain that $x_{0} \leq b$. Hence $b=b \vee x_{0} \prec b \vee x$ by semimodularity. Clearly, $b \vee x \neq u$ since $u \| x$. Moreover, $b \vee x \| u$ since $h(b \vee x)=h(b)+1=h(u)$. Furthermore, $E \cup R_{E}$ is a region (surrounded by $E$ and $\mathcal{B}_{\text {right }}(L)$ ) that contains $b$ and $x$. Hence $b \vee x \in E \cup R_{E}$ since regions are (convex) sublattices. Since $b \vee x \| u \in E$, we obtain that $b \vee x \in R_{E}$. Therefore $b \vee x$ (instead of $x$ ) witnesses that (4.2) holds, which does the job. We have seen that (4.1) holds for each prime interval $[u, v]$.

Finally, for any two atoms, $r_{1}$ and $r_{2},\left\{0, r_{1}, r_{2}, r_{1} \vee r_{2}\right\}$ is a covering square and $\left[0, r_{1}\right] \mu^{*}\left[0, r_{2}\right]$. Hence the lemma follows from (4.1) by transitivity.

The following lemma it not at all surprising.

Lemma 4.5. Let $L$ be a planar lattice, and let $S$ and $T$ be 4 -cells of $D \in \operatorname{Diag}(L)$. Assume that $S$ and $T$ has a common edge on the same side, that is, $\operatorname{Prin}\left(\mathcal{B}_{\text {left }}^{D}(S)\right) \cap$ $\operatorname{Prin}\left(\mathcal{B}_{\text {left }}^{D}(T)\right) \neq \emptyset$ or $\operatorname{Prin}\left(\mathcal{B}_{\text {right }}^{D}(S)\right) \cap \operatorname{Prin}\left(\mathcal{B}_{\text {right }}^{D}(T)\right) \neq \emptyset$. Then $S=T$.

Proof. Assume, by way of contradiction, that $a, b \in L$ such that $a \prec b$ and $a, b \in$ $\mathcal{B}_{\text {left }}(S) \cap \mathcal{B}_{\text {left }}^{D}(T)$. 
Firstly, assume that $a, b \in \mathcal{B}_{\mathrm{sw}}^{D}(S) \cap \mathcal{B}_{\mathrm{sw}}^{D}(T)$. Let $c=w_{S}^{r}=w_{D}^{r}(S)$ and $d=w_{T}^{r}$. Since $S \neq T$, we have that $c \neq d$. Let, say, $d$ be strictly on the right of $c$, and extend $\{a, c, b \vee c\}=\mathcal{B}_{\text {right }}(S)$ to a maximal chain $C$ of $L$. If $b \vee d=1_{T}$ was strictly on the right of $C$, then $b \prec b \vee d$ would contradict (2.5). If $1_{T}$ was strictly on the left of $C$, then $d \prec b \vee d$ would induce the same contradiction. Hence $1_{T} \in C$ together with $h\left(1_{T}\right)=h\left(1_{S}\right)$ gives that $1_{T}=1_{S}$. However, then $c \in \operatorname{int}(T)$ is a contradiction since $T$ is a 4 -cell. By duality, $a, b \in \mathcal{B}_{\text {nw }}(S) \cap \mathcal{B}_{\text {nw }}(T)$ also leads to a contradiction.

Secondly, assume that $a, b \in \mathcal{B}_{\mathrm{sw}}(S) \cap \mathcal{B}_{\mathrm{nw}}(T)$. Let $c=w_{S}^{r}$ again. Since $w_{T}^{r}$ is strictly on the right of the previous $C$ and $b$ is strictly on the left of $C, w_{T}^{r} \prec b$ contradicts (2.5).

Definition 4.6. For $i=1,2$, let $D_{i} \in \operatorname{Diag}\left(L_{i}\right)$, and let $\varphi: L_{1} \rightarrow L_{2}$ be a lattice isomorphism. Then $\varphi$ is a directed diagram isomorphism $\left(L_{1}, D_{1}\right) \rightarrow\left(L_{2}, D_{2}\right)$, if

(i) $\varphi\left(\mathcal{B}_{\text {left }}^{D_{1}}([a, b])\right)=\mathcal{B}_{\text {left }}^{D_{2}}([\varphi(a), \varphi(b)])$ and $\varphi\left(\mathcal{B}_{\text {right }}^{D_{1}}([a, b])\right)=\mathcal{B}_{\text {right }}^{D_{2}}([\varphi(a), \varphi(b)])$, for all $a<b \in L_{1}$, and

(ii) for each maximal chain $C$ of $L_{1}$, we have that $\varphi\left(\operatorname{left}_{\text {side }}^{D_{1}}(C)\right)=\operatorname{left}_{\text {side }}^{D_{2}}(\varphi(C))$ and $\varphi\left(\operatorname{right}_{\text {side }}^{D_{1}}(C)\right)=\operatorname{right}_{\text {side }}^{D_{2}}(\varphi(C))$.

By reflecting the diagram $D$ trough a vertical axis we obtain its mirror image $D^{\text {mir }}$. Let $\operatorname{id}_{L}$ denote the identical $L \rightarrow L, x \mapsto x$ mapping. We say that $L$ is uniquely oriented if for any two planar diagrams $D$ and $F$ of $L, \operatorname{id}_{L}:(L, D) \rightarrow(L, F)$ or $\mathrm{id}_{L}:(L, D) \rightarrow\left(L, F^{\mathrm{mir}}\right)$ is a directed diagram isomorphism.

For example, $S_{7}$ in Figure 2 is uniquely oriented but $M_{3}$ is far from that. We are interested in planar diagram only up to directed diagram isomorphisms.

\section{Lemma 4.7.}

(i) Let $L_{1}$ and $L_{2}$ be glued sum indecomposable slim semimodular lattices, and let $\varphi: L_{1} \rightarrow L_{2}$ be a lattice isomorphism. Assume that $D_{1} \in \operatorname{Diag}\left(L_{1}\right)$ and $D_{2} \in \operatorname{Diag}\left(L_{2}\right)$. Then $\varphi:\left(L_{1}, D_{1}\right) \rightarrow\left(L_{2}, D_{2}\right)$ or $\varphi:\left(L_{1}, D_{1}\right) \rightarrow\left(L_{2}, D_{2}^{\text {mir }}\right)$ is a directed diagram isomorphism.

(ii) Each glued sum indecomposable slim semimodular lattice is uniquely oriented.

Proof. Observe that part (i), applied to the identical mapping, implies part (ii). Hence it suffices to prove part (i). It follows from [7, Lemma 7] that the set $\left\{\varphi\left(\mathcal{B}_{\text {left }}^{D_{1}}\left(L_{1}\right)\right), \varphi\left(\mathcal{B}_{\text {right }}^{D_{1}}\left(L_{1}\right)\right)\right\}$ is equal to $\left\{\mathcal{B}_{\text {left }}^{D_{2}}\left(L_{2}\right), \mathcal{B}_{\text {right }}^{D_{2}}\left(L_{2}\right)\right\}$. Hence, after replacing $D_{2}$ by $D_{2}^{\text {mir }}$ if necessary, we can assume that $\varphi\left(\mathcal{B}_{\text {left }}^{D_{1}}\left(L_{1}\right)\right)=\mathcal{B}_{\text {left }}^{D_{2}}\left(L_{2}\right)$ and $\varphi\left(\mathcal{B}_{\text {right }}^{D_{1}}\left(L_{1}\right)\right)=\mathcal{B}_{\text {right }}^{D_{2}}\left(L_{2}\right)$. For a prime interval $\mathfrak{p} \in \operatorname{Prin}\left(L_{1}\right)$, the distance of $\mathfrak{p}$ from $\mathcal{B}_{\text {left }}^{D_{1}}\left(L_{1}\right)$ will be measured by

$$
d(\mathfrak{p}):=\min \left\{n \in \mathbb{N}_{0}: \text { there is a } \mathfrak{q} \in \operatorname{Prin}\left(\mathcal{B}_{\text {left }}^{D_{1}}\left(L_{1}\right)\right) \text { such that } \mathfrak{q} \mu^{n} \mathfrak{p}\right\},
$$

where $\mu$ is defined right before Lemma 4.4. Notice that, in virtue of (2.3), the covering squares of $L_{1}$ and the 4-cells of $D_{1}$ are the same. Hence $\mu$ in (4.3) can be, and sometimes will be, replaced by $\varrho_{D_{1}}$. For a 4-cell $S$ of $L_{1}$ (with respect to $\left.D_{1}\right)$, we let $d(S):=\min \{d(\mathfrak{p}): \mathfrak{p} \in \operatorname{Prin}(S)\}$. By Lemma $4.4, d(\mathfrak{p})$ and $d(S)$ are well-defined. We will show by induction on $d(S)$ that, for each 4-cell $S$ of $L_{1}$,

$$
\varphi\left(\mathcal{B}_{\text {left }}^{D_{1}}(S)\right)=\mathcal{B}_{\text {left }}^{D_{2}}(\varphi(S)) \text { and } \varphi\left(\mathcal{B}_{\text {right }}^{D_{1}}(S)\right)=\mathcal{B}_{\text {right }}^{D_{2}}(\varphi(S)) .
$$

If $d(S)=0$, which means that $S$ has an edge on the left boundary chain, then (4.4) is evident. Assume that $n:=d(S)>0$, and $\mathfrak{p} \in \operatorname{Prin}(S)$ such that $d(\mathfrak{p})=n$. Then there are a $\mathfrak{q} \in \operatorname{Prin}\left(\mathcal{B}_{\text {left }}^{D_{1}}\left(L_{1}\right)\right)$ and an $\mathfrak{r} \in \operatorname{Prin}\left(L_{1}\right)$ such that $\mathfrak{q} \varrho_{D_{1}}^{n-1} \mathfrak{r}$ and 
$\mathfrak{r} \varrho_{D_{1}} \mathfrak{p}$. Clearly, $d(\mathfrak{r}) \leq n-1$. (Actually, we have equality but we do not need it.) By the definition of $\varrho_{D_{1}}$, there is a 4 -cell $T$ of $L_{1}$ such that $\mathfrak{r}, \mathfrak{p} \in \operatorname{Prin}(T)$. Since $d(T) \leq d(\mathfrak{r}) \leq n-1$, we have that $T \neq S$. The induction hypothesis says that $\varphi\left(\mathcal{B}_{\text {left }}^{D_{1}}(T)\right)=\overline{\mathcal{B}}_{\text {left }}^{D_{2}}(\varphi(T))$ and $\varphi\left(\mathcal{B}_{\text {right }}^{D_{1}}(T)\right)=\mathcal{B}_{\text {right }}^{D_{2}}(\varphi(T))$. By Lemma 4.5 , the common edge $\mathfrak{r}$ of $S$ and $T$ determines how the left-right orientation of $S$ depends on that of $T$, and this "determination" is preserved by $\varphi$. This fact together with the induction hypothesis implies that $\varphi\left(\mathcal{B}_{\text {left }}^{D_{1}}(S)\right)=\mathcal{B}_{\text {left }}^{D_{2}}(\varphi(S))$ and $\varphi\left(\mathcal{B}_{\text {right }}^{D_{1}}(S)\right)=$ $\mathcal{B}_{\text {right }}^{D_{2}}(\varphi(S))$. This completes the proof of $(4.4)$.

In the rest of the proof we will focus mainly on the left sides; if the corresponding right sides are not mentioned then their treatment would be analogous. Let $a<$ $b \in L_{1}$. By Lemma 2.3, we can assume that $[a, b]$ is a glued sum indecomposable lattice since otherwise we could deal with its glued summands. Hence $a$ is meetreducible in $[a, b]$. Moreover, $[a, b]$ is a slim lattice by (2.2). Therefore, we conclude from (2.1) that $a$ has exactly two covers, $c$ and $d$, in $[a, b]$. By semimodularity and (2.3), $S:=\{a, c, d, c \vee d\}$ is a 4-cell. Roughly saying, the idea is that $[a, b]$ is slim, its boundary is determined, its bottom 4-cell $S$ intersects the boundary of $[a, b]$ in $\{a, c, d\}=\mathcal{B}^{D_{1}}(S) \backslash\left\{1_{S}\right\}$, so $S$ determines which one of the boundary chains is the left one and which one is the right one. More exactly, [7, Lemma 7] yields that $\left\{\varphi\left(\mathcal{B}_{\text {left }}^{D_{1}}([a, b])\right), \varphi\left(\mathcal{B}_{\text {right }}^{D_{1}}([a, b])\right)\right\}=\left\{\mathcal{B}_{\text {left }}^{D_{2}}([\varphi(a), \varphi(b)]), \mathcal{B}_{\text {right }}^{D_{2}}([\varphi(a), \varphi(b)])\right\}$. Hence, knowing that (4.4) holds for $S$ and keeping $\{c, d\} \subseteq \mathcal{B}^{D_{1}}([a, b])$ in mind, we conclude that $\varphi$ satisfies $4.6(\mathrm{i})$.

Next, we prove the validity of 4.6 (ii) for $L_{1}$ by induction on $\operatorname{length}\left(L_{1}\right)$. The case length $\left(L_{1}\right)=2$, where $L_{1}$ necessarily equals $2^{2}$, is trivial. Let length $\left(L_{1}\right) \geq 3$, and let $C$ be a maximal chain of $L_{1}$. Since $L_{1}$ is glued sum indecomposable, it has exactly two atoms, $u$ and $v$, by (2.1). Let, say, $u \in C$. We have previously assumed that $\varphi\left(\mathcal{B}_{\text {left }}^{D_{1}}\left(L_{1}\right)\right)=\mathcal{B}_{\text {left }}^{D_{2}}\left(L_{2}\right)$. By the left-right symmetry, we can assume that $u \in \mathcal{B}_{\text {left }}^{D_{1}}\left(L_{1}\right)$; notice that we will not be allowed to use the left-right symmetry for the right side of $C$ later. By the induction hypotheses, $\varphi\left(\operatorname{left}_{\text {side }}^{D_{1} \cap \uparrow u}(C \backslash\{0\})\right)$ is equal to left $\operatorname{side}^{D_{2} \cap \uparrow \varphi(u)}(\varphi(C) \backslash\{0\})$. Hence

$$
\begin{aligned}
\varphi\left(\operatorname{left}_{\text {side }}^{D_{1}}(C)\right) & =\varphi\left(\{0\} \cup \operatorname{left}_{\text {side }}^{D_{1} \cap \uparrow u}(C \backslash\{0\})\right) \\
& =\{0\} \cup \operatorname{left}_{\text {side }}^{D_{2} \cap \uparrow \varphi(u)}(\varphi(C) \backslash\{0\})=\operatorname{left}_{\text {side }}^{D_{2}}(\varphi(C)) .
\end{aligned}
$$

Since $\varphi$ is a bijection, the equation just obtained implies that $\varphi\left(\operatorname{right}_{\text {side }}^{D_{1}}(C)\right)=$ $\varphi\left(C \cup\left(L_{1} \backslash \operatorname{left}_{\text {side }}^{D_{1}}(C)\right)\right)=\varphi(C) \cup\left(L_{2} \backslash \operatorname{left}_{\text {side }}^{D_{2}}(\varphi(C))\right)=\operatorname{right}_{\text {side }}^{D_{2}}(\varphi(C))$. This shows that $\varphi$ satisfies $4.6($ ii), completing the induction.

Let $L$ be a planar semimodular lattice, and $x \in L$. We say that $x$ is a possible weak corner of $L$, if $x$ is a weak corner of $L$ with respect to some planar diagram of $L$. The set of possible weak corners of $L$ will be denoted by $\operatorname{Corn}_{\mathrm{pw}}(L)$. Clearly, $\operatorname{Corn}_{\mathrm{pw}}(L) \subseteq \operatorname{Ji}(L) \cap \operatorname{Mi}(L)$ but the converse inclusion does not hold in general. (For example, if $L$ is obtained from a grid by insert an eye $e$ into a "middle 4-cell", then $e \notin \operatorname{Corn}_{\mathrm{pw}}(L)$.) The set of non-chain intervals of length 2 will be denoted by $\operatorname{Ivl}_{2}(L)$. By the trunk of an $I \in \operatorname{Ivl}_{2}(L)$, denoted by $\operatorname{Trnk}(I)$, we mean the nontrivial antichain $I \backslash\left\{0_{I}, 1_{I}\right\}$. As usual, the unique lower cover and upper cover of a doubly irreducible element $x$ is denoted by $x^{-}$and $x^{+}$, respectively.

Lemma 4.8. Let $L$ be a glued sum indecomposable planar semimodular lattice with a fixed planar diagram D. Then $\operatorname{Corn}_{\mathrm{pw}}(L)=\{x \in L: x$ is a double irreducible 
element, $\left[x^{-}, x^{+}\right] \in \operatorname{Ivl}_{2}(L)$, and $\operatorname{Trnk}\left(\left[x^{-}, x^{+}\right]\right)$contains a weak corner of $L$ with respect to $D\}$.

Proof. Let $U$ denote the set on the left of the equality sign in the lemma. Firstly, to prove the " $\supseteq$ " inclusion, assume that $x \in U$. Let $w$ be a weak corner of $D$ witnessing that $x \in U$. Clearly, $\left[w^{-}, w^{+}\right]=\left[x^{-}, x^{+}\right]$. Since both $x$ and $w$ are doubly irreducible elements of $\left[w^{-}, w^{+}\right] \in \operatorname{Ivl}_{2}(L)$, there is an automorphism of $L$ that interchanges $w$ and $x$ but keeps the rest of elements fixed. Therefore, if we interchange the labels $w$ and $x$ in the diagram $D$, we obtain a new diagram in which $x$ is a weak corner. Hence $x \in \operatorname{Corn}_{\mathrm{pw}}(L)$, which proves that $\operatorname{Corn}_{\mathrm{pw}}(L) \supseteq U$.

To prove the converse inclusion, assume that $v \in \operatorname{Corn}_{\mathrm{pw}}(L)$. Then there is an $F \in \operatorname{Diag}(L)$ such that $v$ is a weak corner with respect to $F$. This implies that $v \in \operatorname{Ji}(L) \cap \operatorname{Mi}(L)$. We obtain from Lemma 4.3 that $v^{-}$has a cover $y$ that is distinct from $v$. Since $v=v \vee v^{-} \preceq v \vee y \neq v$ by semimodularity and $v^{+}$is the only cover of $v$, we obtain that $v \vee y=v^{+}$and $I:=\left[v^{-}, v^{+}\right] \in \operatorname{Ivl}_{2}(L)$. This yields that if $v$ happens to be a weak corner of $D$, then $v \in U$. So we can assume that $v$ is not a weak corner of $D$. Let $L_{D}^{\prime}$ be the full slimming sublattice of $L$ with respect to $D$. Assume for a contradiction that $v \in L_{D}^{\prime}$. Then $v$ does not belong to $\operatorname{int}^{D}\left(L_{D}^{\prime}\right)$ since otherwise it would be join-reducible by (2.10). Hence $v \in \mathcal{B}^{D}\left(L_{D}^{\prime}\right)=\mathcal{B}^{D}(L)$, implying that $v$ is a weak corner of $D$, a contradiction again.

Therefore, $v \in L \backslash L_{D}^{\prime}$. Let $a$ resp. $b$ denote the left weak corner resp. the right weak corner of $I$ with respect to $D$. In other words, $a$ is the leftmost element of $\operatorname{Trnk}(I)$, and $b$ is the rightmost one. Clearly, $a, b \in L_{D}^{\prime}$. Hence $|\{v, a, b\}|=3$. It follows from $v \in \mathcal{B}^{F}(L)$, (2.6) and (2.9) that $v$ belongs to $\mathcal{B}^{F}(I)$. This together with $\left|\mathcal{B}^{F}(I)\right| \leq 2$ and $|\{v, a, b\}|=3$ yields that $\{a, b\} \nsubseteq \mathcal{B}^{F}(I)$. Let, say, $b \notin \mathcal{B}^{F}(I)$. We conclude from (2.7) that $v^{-}$is the only lower cover of $b$ and $v^{+}$is the only upper cover of $b$. Hence $b \in \operatorname{Ji}(L) \cap \operatorname{Mi}(L)$. In particular, $b \in \operatorname{Ji}\left(L_{D}^{\prime}\right)$. Hence (2.10) implies that $b \in \mathcal{B}^{D}\left(L_{D}^{\prime}\right)=\mathcal{B}^{D}(L)$. Therefore, $b$ is a weak corner of $D$. Thus, $v \in U$.

Lemma 4.9. Let $L$ be a rectangular lattice, and let $F$ be a planar diagram of $L$. (Not necessarily the same that witnesses the rectangularity of L.) Then

(i) F has exactly one left weak corner $w_{F}^{\ell}$ and exactly one right weak corner $w_{F}^{r}$, and they are complementary.

(ii) Consequently, all planar diagrams are "equally appropriate" when we want to verify the rectangularity of a planar semimodular lattice.

(iii) $\mathcal{B}^{F}(L) \backslash \mathcal{B}_{\text {south }}^{F}(L)$ and $\mathcal{B}^{F}(L) \backslash \mathcal{B}_{\text {north }}^{F}(L)$ do not depend on $F \in \operatorname{Diag}(L)$.

Notice that 4.9(ii) will often be used implicitly.

Proof. We can assume that length $(L) \geq 3$ since otherwise the statement is evident. Let $D$ be a fixed planar diagram that witnesses the rectangularity of $L$. In particular, we know that $w_{D}^{\ell}$ and $w_{D}^{r}$ are complementary elements. Let $I_{\ell}^{D}:=\left[w_{D}^{\ell}{ }^{-}, w_{D}^{\ell}{ }^{+}\right]$ and $I_{r}^{D}:=\left[w_{D}^{r-}, w_{D}^{r+}\right]$ be the intervals of length 2 whose trunk contains $w_{D}^{\ell}$ and $w_{D}^{r}$, respectively.

Next, let $F \in \operatorname{Diag}(L)$ be arbitrary. We know from (2.4) that there is a double irreducible element $x$ in $\mathcal{B}_{\text {left }}^{F}(L) \backslash\{0,1\}$. Lemma 4.8 implies that $x \in \operatorname{Trnk}\left(I_{\ell}^{D}\right)$ or $x \in \operatorname{Trnk}\left(I_{r}^{D}\right)$. Let, say $x \in \operatorname{Trnk}\left(I_{\ell}^{D}\right)$; the other case would be left-right symmetric and needs no separate treatment.

Assume for a contradiction that $x^{\prime}$ is another weak corner of $F$ such that $x^{\prime}$ is comparable with $x$. Let, say, $x^{\prime}<x$. Since $x^{\prime+} \neq x$ by Lemma 4.3, we have that 
$x^{\prime+} \leq x^{-}$. We obtain $x^{\prime} \in \operatorname{Trnk}\left(I_{r}^{D}\right)$ from Lemma 4.8 , and $w_{D}^{r+}=x^{++} \leq x^{-}=$ $w_{D}^{\ell}{ }^{-}$. Hence $w_{D}^{r}<w_{D}^{\ell}$ contradicts the fact that $w_{D}^{\ell}$ and $w_{D}^{\ell}$ are complementary elements and they are distinct from 0 and 1 . Consequently,

no two distinct weak corners of $F$ are comparable.

In particular, since $\mathcal{B}_{\text {left }}^{F}(L)$ is a chain, $x$ is the only weak left corner of $F$; so we denote it by $x=w_{F}^{\ell}$. Clearly,

$$
w_{F}^{\ell-}=w_{D}^{\ell-} \text { and } w_{F}^{\ell^{+}}=w_{D}^{\ell+} .
$$

Similarly, $\mathcal{B}_{\text {right }}^{F}(L)$ has a unique doubly irreducible element $y$, and $y \in \operatorname{Trnk}\left(I_{\ell}^{D}\right)$ or $y \in \operatorname{Trnk}\left(I_{r}^{D}\right)$. Assume for a contradiction that $y \in \operatorname{Trnk}\left(I_{\ell}^{D}\right)$. Then $y^{-}=$ $\left(w_{D}^{\ell}\right)^{-}=x^{-}$and $y^{+}=\left(w_{D}^{\ell}\right)^{+}=x^{+}$. Since $x^{-} \in \mathcal{B}_{\text {left }}^{F}(L), x^{-}=y^{-} \in \mathcal{B}_{\text {right }}^{F}(L)$ and $L$ is glued sum indecomposable, it follows that $x^{-}=0_{L}$. Dually, $x^{+}=y^{+} \in$ $\mathcal{B}_{\text {left }}^{F}(L) \cap \mathcal{B}_{\text {right }}^{F}(L)$ yields that $x^{+}=1_{L}$. This contradicts length $(L) \geq 3$. Therefore $y=w_{F}^{r} \in \operatorname{Trnk}\left(I_{r}^{D}\right)$ is the unique right weak corner of $F$ and we have that

$$
w_{F}^{r-}=w_{D}^{r-} \quad \text { and } \quad w_{F}^{r+}=w_{D}^{r+} .
$$

We know from (4.5) that $w_{F}^{\ell} \| w_{F}^{r}$. Hence (4.6) and (4.7) yield that $w_{F}^{\ell} \wedge w_{F}^{r}=$ $w_{F}^{\ell^{-}} \wedge w_{F}^{r-}=w_{D}^{\ell-} \wedge w_{D}^{r-}=0$ and $w_{F}^{\ell} \vee w_{F}^{r}=w_{F}^{\ell+} \vee w_{F}^{r+}=w_{D}^{\ell+} \vee w_{D}^{r+}=1$. That is, $w_{F}^{\ell}$ and $w_{F}^{r}$ are complementary elements. This proves 4.9(i).

Next, (2.12) yields that

$$
\mathcal{B}^{F}(L) \backslash \mathcal{B}_{\text {south }}^{F}(L)=\uparrow w_{F}^{\ell+} \cup \uparrow w_{F}^{r+} \text { and } \mathcal{B}^{F}(L) \backslash \mathcal{B}_{\text {north }}^{F}(L)=\downarrow w_{F}^{\ell-} \cup \downarrow w_{F}^{r-} \text {, }
$$
proving 4.9(iii). Finally, (4.8), (4.6) and (4.7) imply 4.9(ii).

\section{Some Properties of ElEMENTS AND ReCtangular intervals Versus} DIAGRAMS

Lemma 5.1. Let $I$ be a rectangular interval of a slim semimodular lattice L. Assume that $a \in I, b \in L \backslash I$ such that $a<b$. Then $[a, b] \cap \mathcal{B}_{\text {north }}^{D}(L) \neq \emptyset$, for all $D \in \operatorname{Diag}(L)$.

Proof. Let $D \in \operatorname{Diag}(L)$. We know from Lemma 4.9(ii) that the rectangularity of $I$ is witnessed by $D$. It follows from (2.6) and (2.7) that there is a maximal $x \in \mathcal{B}(I)=\mathcal{B}^{D}(I)$ such that $a \leq x<b$. Let $x^{+}$be an atom in $[x, b]$; it is not in $I$ by the choice of $x$. If we had $x \notin \mathcal{B}_{\text {north }}(I)$, then $x$ would have at least two additional covers in $I$ by (2.14), which would contradict (2.1). Hence $x \in[a, b] \cap \mathcal{B}_{\text {north }}(I)$ proves the statement.

Lemma 5.2. Assume that $I$ is a rectangular interval of a slim semimodular lattice $L$ and $D \in \operatorname{Diag}(L)$. Then the intervals $\left[0_{I}, w_{D}^{\ell}(I)\right]_{L}$ and $\left[0_{I}, w_{D}^{r}(I)\right]_{L}$, understood in L, are chains.

Proof. By way of contradiction, we assume that, say, $\left[0_{I}, w_{I}^{\ell}\right]_{L}$ is not a chain. Then there is an $x \in\left[0_{I}, w_{I}^{\ell}\right]_{L} \backslash\left\{w_{I}^{\ell}\right\}$ with (at least) two distinct covers, $y_{1}$ and $y_{2}$, in $\left[0_{I}, w_{I}^{\ell}\right]_{L}$. Let, say, $y_{1} \in\left[0_{I}, w_{I}^{\ell}\right]_{I}$ (the interval within $I$ ). Then $y_{2} \notin I$, since $\left[0_{I}, w_{I}^{\ell}\right]_{I}$ is a chain by (2.11) and (2.12). By (2.14), there is an $y_{3} \in I \backslash\left\{y_{1}\right\}$ such that $x \prec y_{3}$. Now we have three distinct covers of $x$, which contradicts (2.1).

Lemma 5.3. If $L$ is a planar semimodular lattice and $u \in L \backslash \operatorname{Mi}(L)$, then any two covers of $u$ have the same join. 
Proof. Fix a $D \in \operatorname{Diag}(L)$, and let $L^{\prime}$ be the full slimming sublattice of $L$ with respect to $D$. Then $u \in L^{\prime}$. We obtain from (2.1) that $u$ has exactly two covers, $a$ and $b$, within $L^{\prime}$. Let $v=a \vee b \in L^{\prime}$. All further covers of $u$ in $L$ are eyes belonging to $[u, v]$. Hence the join of arbitrary two distinct covers of $u$ equals $v$.

If $L$ is a planar semimodular lattice and $x \in L$, then the height of $x$ is defined to be the length of any maximal chain of $\downarrow x$. (By the Jordan-Hölder theorem, no matter which chain is considered). Let $D \in \operatorname{Diag}(L)$ be fixed. Let $x, y \in L$ with $h(x)=h(y)$. We say that $x$ is on the left of $y$, with respect to $D$, if for every (equivalently, some) maximal chain $C$ of $L$ that contains $y, x$ is on the left of $C$. (Equivalently, if $y \in \operatorname{right}_{\text {side }}^{D}(C)$ for all maximal chains $C$ that contain $x$.) Let us emphasize that, in our terminology, " $x$ is on the left of $y$ " implies that $h(x)=h(y)$. If $x$ is on the left of $y, x \neq y$ and there is no $z \in L \backslash\{x, y\}$ such that $x$ is on the left of $z$ and $z$ is on the left of $y$, then $y$ is the right neighbor of $x$ (with respect to $D)$. Clearly,

$$
\begin{aligned}
& \text { if } x \text { belongs a maximal chain } C, h(x)=h(y) \text { and } y \text { is (strictly) } \\
& \text { on the left of } x \text {, then } y \text { is (strictly) on the left of } C \text {; } \\
& \text { if } x \text { is on the left of } y, y \text { is on the left of } x \text { and } h(x)=h(y) \text {, then } x=y \text {; } \\
& \text { if } x \in \mathcal{B}_{\text {left }}(L) \text { and } h(x)=h(y) \text {, then } x \text { is on the left of } y \text {; } \\
& \text { each } x \in L \backslash \mathcal{B}_{\text {right }}^{D}(L) \text { has a unique right neighbor (with respect to } D \text { ). }
\end{aligned}
$$

Notice that these assertions imply, for a planar semimodular $L$, that

$$
\text { if } a \in \mathcal{B}_{\text {left }}^{D}(L) \text {, then } \mathcal{B}_{\text {left }}^{D}(\downarrow a)=\mathcal{B}_{\text {left }}^{D}(L) \cap \downarrow a \text { and } \mathcal{B}_{\text {left }}^{D}(\uparrow a)=\mathcal{B}_{\text {left }}^{D}(L) \cap \uparrow a \text {. }
$$

Indeed, for $x \in \mathcal{B}_{\text {left }}^{D}(L) \cap \downarrow a$, let $y$ denote the unique element of $\mathcal{B}_{\text {left }}^{D}(\downarrow a)$ such that $h(x)=h(y)$. Applying (5.3) to $L$ and also to $\downarrow a$, we obtain that $x$ and $y$ are mutually on the left of each other. Hence they are equal by (5.2), and the first equality of (5.5) follows. The second one holds by duality.

The following lemma is the counterpart of Lemma 5.3. Although it looks evident by our geometric intuition, its rigorous proof needs a result borrowed from Kelly and Rival [23].

Lemma 5.4. Assume that $a$ and $b$ are the leftmost lower cover and the rightmost lower cover of an element $v$ in some planar diagram of a planar lattice $L$, respectively. Then $a \wedge b$ is the meet of all lower covers of $v$.

Proof. We can clearly assume that $a \neq b$. Let $u=a \wedge b$. By (2.6), $I:=[u, v]$ is a region. Let $C_{0}$ and $C_{1}$ be maximal chains in $\downarrow u$ and in $\uparrow v$, respectively. Then $W:=C_{0} \cup \mathcal{B}_{\text {left }}(I) \cup C_{1}$ and $E:=C_{0} \cup \mathcal{B}_{\text {right }}(I) \cup C_{1}$ are maximal chains in $L$. It follows from Kelly and Rival [23] that

$$
\begin{gathered}
I=\{x \in L: x \text { is on the right of } W, x \text { is and on the left of } E, \\
x \not \leq u \text { and } x \geq v\} .
\end{gathered}
$$

Let $x$ be a lower cover of $v$. Then $x$ cannot be strictly on the left of $W$ since then $x$ would be strictly on the left of the leftmost lower cover, $a$. Hence $x$ is on the right of $W$ and, similarly, on the left of $E$. This together with (5.6) shows that $x \in I$. Hence $u=0_{I} \leq x$ for all lower covers $x$ of $v$, proving the lemma.

Although the boundary of a planar semimodular lattice $L$ is not unique in general, see $M_{3}$, the following assertion holds. 
Lemma 5.5. Let $L$ be a glued sum indecomposable planar semimodular lattice, and let $D$ be a planar diagram of $L$. Let $I$ and $J$ be rectangular intervals such that $I \cap J$ is a chain. Then $I \cap J \subseteq \mathcal{B}^{D}(I) \cap \mathcal{B}^{D}(J)$.

Proof. Let $x$ be the least element of the chain $I \cap J$. Assume first that $x \in \operatorname{int}^{D}(I) \cap$ $\operatorname{int}^{D}(J)$. Then $x \neq 0_{L}$, so $x$ has a lower cover $y$. By (2.6) and (2.8), $y \in I \cap J$, contradicting the choice of $x$. This excludes that $x \in \operatorname{int}^{D}(I) \cap \operatorname{int}^{D}(J)$.

Secondly, we assume that $x \in \mathcal{B}^{D}(I) \cap \operatorname{int}^{D}(J)$. By (2.6) and (2.8), all lower covers of $x$ belong to $J$. Hence, by the choice of $x$, no lower cover of $x$ belongs to $I$. This means that $x=0_{I}$. However, then $x$ has at least two covers in $I$, and these covers belong to $J$ (and therefore to $I \cap J$ ) by (2.6) and (2.8). This is a contradiction since $I \cap J$ is a chain. Thus, taking the $I-J$ symmetry into account, we conclude that $x \notin \mathcal{B}^{D}(I) \cap \operatorname{int}^{D}(J)$ and $x \notin \operatorname{int}^{D}(I) \cap \mathcal{B}^{D}(J)$.

Therefore, $x \in \mathcal{B}^{D}(I) \cap \mathcal{B}^{D}(J)$. Assume for a contradiction that $x$ has more than one covers both in $I$ and $J$. Let $a_{1}, a_{2} \in I$ and $b_{1}, b_{2} \in J$ covers of $x$ such that $a_{1} \neq a_{2}$ and $b_{1} \neq b_{2}$. Let $v:=a_{1} \vee a_{2} \in I$. Since $a_{1} \vee a_{2}=b_{1} \vee b_{2}$ by Lemma 5.3, $v \in J$. By the convexity of $I \cap J$, we have that $\left\{a_{1}, a_{2}\right\} \subseteq[u, v] \subseteq I \cap J$, which is a contradiction since $I \cap J$ is a chain. This proves that, say, $x$ has at most one cover in $I$. This fact together with (2.14) implies that $x \in \mathcal{B}_{\text {north }}^{D}(I)$.

We are now in the position to show that each $y \in I \cap J$ belongs to $\mathcal{B}^{D}(I) \cap \mathcal{B}^{D}(J)$. We already know this if $y=x$. Hence we can assume that $y>x$. We obtain from $x \in \mathcal{B}_{\text {north }}^{D}(I)$ and (2.12) that $y \in \mathcal{B}_{\text {north }}^{D}(I) \backslash \mathcal{B}_{\text {south }}^{D}(I)$. Hence (2.15) yields that $y$ has at least two lower covers, $z_{1}$ and $z_{2}$, in $I$. If we had $y \in \operatorname{int}^{D}(J)$, then $(2.6)$ and (2.8) would imply $\left\{z_{1}, z_{2}\right\} \subseteq J$, and the antichain $\left\{z_{1}, z_{2}\right\} \subseteq I \cap J$ would be a contradiction. Consequently, $y \in \mathcal{B}^{D}(J)$, whence $y \in \mathcal{B}^{D}(I) \cap \overline{\mathcal{B}}^{D}(J)$ proves the statement.

\section{Some properties that Depend on the Full Slimming of $L$}

Given a rectangular interval or, in particular, a patch (interval) $I=[u, v]$ of $L$, its bottom and top will sometimes be denoted by $0_{I}=u$ and $1_{I}=v$, while $w_{I}^{\ell}=w_{D}^{\ell}(I)$ and $w_{I}^{r}=w_{D}^{r}(I)$ stand for its weak corners, with respect to $D \in \operatorname{Diag}(L)$.

Lemma 6.1. Let $L$ be a planar semimodular lattice, and let $L^{\prime}$ be the full slimming of $L$. Then $L^{\prime}$ is a slim semimodular lattice, and the following five assertions hold.

(i) $L$ is a patchwork-irreducible lattice iff so is $L^{\prime}$;

(ii) $L$ is a rectangular lattice iff so is $L^{\prime}$;

(iii) $L$ is a patch lattice (that is, a rectangular lattice whose weak corners are coatoms, see also Lemma 4.9(ii)) iff so is $L^{\prime}$;

(iv) $L$ is glued sum indecomposable iff so is $L^{\prime}$.

(v) $L$ is indecomposable with respect to the Hall-Dilworth gluing over chains iff so is $L^{\prime}$.

Moreover, if $D$ is a fixed planar diagram of $L, L^{\prime}$ denotes the full slimming sublattice of $L$ with respect to $D$, and $D^{\prime}$ is the restriction of $D$ to $L^{\prime}$, then the following three assertions also hold.

(vi) $\mathcal{B}^{D}(L)=\mathcal{B}^{D^{\prime}}\left(L^{\prime}\right)$.

(vii) Let $a<b \in L$. Then $[a, b]_{L}$ is a rectangular interval of $L$ iff $a, b \in L^{\prime}$ and $[a, b]_{L^{\prime}}$ is a rectangular interval of $L^{\prime}$. In particular, $[a, b]_{L} \in \mathcal{P}(L)$ iff $a, b \in L^{\prime}$ and $[a, b]_{L^{\prime}} \in \mathcal{P}\left(L^{\prime}\right)$. Hence $[a, b]_{L} \in \mathcal{P}_{\max }(L)$ iff $a, b \in L^{\prime}$ and $[a, b]_{L^{\prime}} \in \mathcal{P}_{\max }\left(L^{\prime}\right)$. 
(viii) Let $\mathcal{H}=\left\{\left[a_{i}, b_{i}\right]_{L}: 1 \leq i \leq n\right\}$ be a system of rectangular intervals of $L$, and let $\boldsymbol{H}^{\prime}=\left\{\left[a_{i}, b_{i}\right]_{L^{\prime}}: 1 \leq i \leq n\right\}$ be the corresponding system of rectangular intervals of $L^{\prime}$, see part (vii). Then $\mathcal{H}$ is a patchwork system for $D$ iff $\boldsymbol{\mathcal { H }}^{\prime}$ is a patchwork system for $D^{\prime}$.

In connection with parts (vi)-(viii), notice that we often write $D$ for $D^{\prime}$ in the paper when an interval or a sublattice of $L$ is considered.

Proof. In virtue of Lemma 4.1 (see also Remark 4.2), we can assume that $L^{\prime}$ is the full slimming sublattice of $L$ with respect to a fixed planar diagram $D$ even in parts (ii)-(v) of the lemma. We will have to be more careful in case of (i) since the full slimming sublattice and the patchwork system may depend on different diagrams. Similarly, $L$ could have intervals $I$ whose rectangularity comes from diagrams distinct from the restriction of $D$ to $I$, and this phenomenon would cause a lot of difficulty while proving (vii). Fortunately, Lemma 4.9 allows us to use $D$ (and its restriction) without caring with this possibility.

We know from Proposition 2.1 that $L^{\prime}$ is a slim semimodular lattice. We can assume that $L \neq L^{\prime}$. Let $e \in L \backslash L^{\prime}$ denote an arbitrary eye. The 4-cell $\left\{e^{-}, a, b, e^{+}\right\}$ of $L^{\prime}$, see (2.16), will be denoted by $S$. The notation $a=a_{e}$ and $b=b_{e}$ are also fixed in the proof.

Since $e \in \operatorname{int}^{D^{\prime}}(S) \subseteq \operatorname{int}^{D^{\prime}}\left(L^{\prime}\right)$ by (2.9), we insert the eyes into the interior of $L^{\prime}$. Hence $\mathcal{B}^{D}(L)=\mathcal{B}^{D^{\prime}}\left(L^{\prime}\right)$. This gives (vi), which implies (ii) and (iii). We also obtain (iv) since glued sum indecomposability in the planar case means that the lattice in question has at least four elements and $\{0,1\}$ is the intersection of the left and the right boundary chains.

Assume that an eye $e$ belongs to a rectangular interval $I$. Since $e \in \operatorname{Mi}(L) \cap \operatorname{Ji}(L)$, we obtain that $e \notin\left\{0_{I}, 1_{I}\right\}$. Hence $\left[e^{-}, e^{+}\right] \subseteq I$. Using (2.9), we conclude that $e \in \operatorname{int}^{D}\left(\left[e^{-}, e^{+}\right]\right) \subseteq \operatorname{int}^{D}(I)$. That is,

$e$ cannot be on the boundary of a rectangular interval of $L$.

In particular, (6.1) implies that an eye cannot be the bottom or the top of a rectangular interval. Therefore, if we consider an interval $I$ as the pair $\left(0_{I}, 1_{I}\right)$, then we can say that $L^{\prime}$ and $L$ has "exactly the same" rectangular intervals. This implies the first half (vii). The rest of (vii) is then evident since $L^{\prime}$ is a coverpreserving sublattice of $L$.

While proving (viii), we use the following notation: for $I \in \mathcal{H}$, we let $I^{\prime}:=$ $I \cap L^{\prime}=\left[0_{I}, 1_{I}\right]_{L^{\prime}}$; and for $J^{\prime} \in \mathcal{H}^{\prime}$, we let $J:=\left[0_{J^{\prime}}, 1_{J^{\prime}}\right]_{L}$. By the definition of $\mathcal{H}^{\prime}$, we have that

$$
K \in \mathcal{H} \text { iff } K^{\prime} \in \mathcal{H}^{\prime}, \text { and }(I, J) \in \mathcal{E}(\mathcal{H}) \text { iff }\left(I^{\prime}, J^{\prime}\right) \in \mathcal{E}\left(\mathcal{H}^{\prime}\right) .
$$

Since the tops and the bottoms of covering squares are the same in $L$ as in $L^{\prime},(6.2)$ yields that $\mathcal{H}$ satisfies 3.1 (i) iff so does $\boldsymbol{H}^{\prime}$.

If $\mathcal{H}$ satisfies 3.1(ii), then so does $\mathcal{H}^{\prime}$, evidently. Before proving the converse implication, we we assert that, for all $I, J \in \mathcal{H}$,

$$
\text { if } I^{\prime} \cap J^{\prime} \text { is a chain, then } I \cap J=I^{\prime} \cap J^{\prime} \text {, whence } I \cap J \text { is also a chain. }
$$

By way of contradiction, let us assume that (6.3) fails for some $I, J \in \mathcal{H}$. Then $I \cap J=\left[0_{I} \vee 0_{J}, 1_{I} \wedge 1_{J}\right]_{L}$ contains an eye $e$. If $0_{I} \vee 0_{J} \notin\left\{0_{I}, 0_{J}\right\}$, then $0_{I} \vee 0_{J}$ is join-reducible. If $0_{I} \vee 0_{J} \in\left\{0_{I}, 0_{J}\right\}$, then $0_{I} \vee 0_{J}$ is meet-reducible. Hence, in both cases, $0_{I} \vee 0_{J} \notin \operatorname{Ji}(L) \cap \operatorname{Mi}(L)$. Dually, $1_{I} \wedge 1_{J} \notin \operatorname{Ji}(L) \cap \operatorname{Mi}(L)$. Therefore, 
$e \notin\left\{0_{I} \vee 0_{J}, 1_{I} \wedge 1_{J}\right\}$. Hence $0_{I} \vee 0_{J}<e<1_{I} \wedge 1_{J}$, and we conclude that $0_{I} \vee 0_{J} \leq e^{-}<a<e^{+} \leq 1_{I} \wedge 1_{J}$. This yields that $a \in I^{\prime} \cap J^{\prime}$. We obtain $b \in I^{\prime} \cap J^{\prime}$ similarly, which is a contradiction since $I^{\prime} \cap J^{\prime}$ is a chain. This proves (6.3).

Next, assume that $\boldsymbol{H}^{\prime}$ satisfies $3.1\left(\right.$ ii), and let $(I, J) \in \mathcal{E}(\boldsymbol{H})$. Then $\left(I^{\prime}, J^{\prime}\right) \in$ $\mathcal{E}\left(\mathcal{H}^{\prime}\right)$ by $(6.2)$, whence $I^{\prime} \cap J^{\prime}$ is a chain. So is $I \cap J$ by (6.3). Hence $\mathcal{H}$ also satisfies 3.1(ii).

As a preparation for 3.1(iii), assume that $K^{\prime}$ is an arbitrary rectangular interval of $L^{\prime}$. Equivalently, see 6.1(vii), we assume that $K$ is a rectangular interval of $L$. We claim that

$$
\begin{aligned}
& K^{\prime} \text { is the full slimming sublattice of } K \\
& \text { with respect to (the restriction of) } D \text {. }
\end{aligned}
$$

It suffices to show that if $K$ contains an eye $e \in L \backslash L^{\prime}$, then $\left\{e^{-}, e^{+}\right\} \subseteq K^{\prime}$. But this is easy: if $0_{K} \leq e \leq 1_{K}$, then $0_{K}<e<1_{K}$ since $e \in \operatorname{Ji}(L) \cap \operatorname{Mi}(L), 0_{K} \notin \operatorname{Mi}(L)$ and $1_{K} \notin \operatorname{Ji}(L)$, whence $0_{K} \leq e^{-}<e<e^{+} \leq 1_{K}$, indeed.

Since $D^{\prime}$ is a restriction of $D$, either of (6.1) and (6.4) yields that

$$
\mathcal{B}_{\text {left }}^{D^{\prime}}\left(K^{\prime}\right)=\mathcal{B}_{\text {left }}^{D}(K) \text { and } \mathcal{B}_{\text {right }}^{D^{\prime}}\left(K^{\prime}\right)=\mathcal{B}_{\text {right }}^{D}(K) .
$$

It follows from (2.13), (2.14) and (2.15) that, for every rectangular lattice $R$ and $F \in \operatorname{Diag}(R)$,

$$
\mathcal{B}_{\text {north }}^{F}(R)=\mathcal{B}^{F}(R) \backslash(R \backslash \operatorname{Mi}(R)) \text { and } \mathcal{B}_{\text {south }}^{F}(R)=\mathcal{B}^{F}(R) \backslash(R \backslash \operatorname{Ji}(R)) .
$$

Furthermore, it follows from (6.4) that $K^{\prime} \backslash \operatorname{Mi}\left(K^{\prime}\right)=K \backslash \operatorname{Mi}(K)$ and $K^{\prime} \backslash \operatorname{Ji}\left(K^{\prime}\right)=$ $K \backslash \operatorname{Ji}(K)$. This together with (6.5) and (6.6) yields that

$$
\mathcal{B}_{\text {north }}^{D^{\prime}}\left(K^{\prime}\right)=\mathcal{B}_{\text {north }}^{D}(K) \text { and } \mathcal{B}_{\text {south }}^{D^{\prime}}\left(K^{\prime}\right)=\mathcal{B}_{\text {south }}^{D}(K) \text {. }
$$

Next, assume that $\mathcal{H}$ is a patchwork system for $D$. We have already seen that $\mathcal{H}^{\prime}$ satisfies $3.1(\mathrm{i})$ and $3.1(\mathrm{ii})$. Let $\left(I^{\prime}, J^{\prime}\right) \in \mathcal{E}\left(\boldsymbol{H}^{\prime}\right)$. Since $(I, J) \in \mathcal{E}(\mathcal{H})$ by $(6.2)$, we have that, say, $I \cap J \subseteq \mathcal{B}_{\text {north }}^{D}(I) \cap \mathcal{B}_{\text {south }}^{D}(J)$. Hence, using (6.7), we obtain that $I^{\prime} \cap J^{\prime} \subseteq I \cap J \subseteq \mathcal{B}_{\text {north }}^{D}(I) \cap \mathcal{B}_{\text {south }}^{D}(J)=\mathcal{B}_{\text {north }}^{D^{\prime}}\left(I^{\prime}\right) \cap \mathcal{B}_{\text {south }}^{D^{\prime}}\left(J^{\prime}\right)$. This shows that $\mathcal{H}^{\prime}$ also satisfies 3.1(iii) for $D^{\prime}$, so it is a patchwork system for $D^{\prime}$.

Conversely, assume that $\mathcal{H}^{\prime}$ is a patchwork system for $D^{\prime}$. We have already seen that $\mathcal{H}$ satisfies $3.1(\mathrm{i})$ and $3.1(\mathrm{ii})$. Let $(I, J) \in \mathcal{E}(\boldsymbol{H})$. Since $\left(I^{\prime}, J^{\prime}\right) \in \mathcal{E}\left(\mathcal{H}^{\prime}\right)$ by (6.2), we have that $I^{\prime} \cap J^{\prime}$ is a chain and, say, $I^{\prime} \cap J^{\prime} \subseteq \mathcal{B}_{\text {north }}^{D^{\prime}}\left(I^{\prime}\right) \cap \mathcal{B}_{\text {south }}^{D^{\prime}}\left(J^{\prime}\right)$. Consequently, using (6.3) and (6.7), we obtain that $I \cap J=I^{\prime} \cap J^{\prime} \subseteq \mathcal{B}_{\text {north }}^{D^{\prime}}\left(I^{\prime}\right) \cap$ $\mathcal{B}_{\text {south }}^{D^{\prime}}\left(J^{\prime}\right)=\mathcal{B}_{\text {north }}^{D}(I) \cap \mathcal{B}_{\text {south }}^{D}(J)$. Hence $\mathcal{H}$ satisfies 3.1 (iii) for $D$. Thus, it is a patchwork system for $D$. This completes the proof of (viii).

Next, armed with (viii), we derive (i). Assume that $L$ is patchwork-reducible. Then there is a $D \in \operatorname{Diag}(L)$ and there is a nontrivial patchwork system $\mathcal{H}$ for $D$. Let $L_{D}^{\prime}$ be the full slimming sublattice of $L$ with respect to $D$. We conclude from (viii) that $\mathcal{H}^{\prime}=\mathcal{H}_{D}^{\prime}$ is a nontrivial patchwork system for (the diagram restricted to) $L_{D}^{\prime}$. Hence $L_{D}^{\prime}$ is patchwork-reducible, and so is $L^{\prime}$ since $L^{\prime} \cong L_{D}^{\prime}$ by Lemma 4.1.

Conversely, assume that $L^{\prime}$ is patchwork-reducible. Hence there is a $D^{\prime}$ in $\operatorname{Diag}\left(L^{\prime}\right)$ such that there is a nontrivial patchwork system $\mathcal{H}^{\prime}$ for $D^{\prime}$. Let $F \in$ $\operatorname{Diag}(L)$, and take the full slimming sublattice $L_{0}$ of $L$ determined by $F$. Clearly, $F^{\text {mir }}$ would determine the same full slimming sublattice $L_{0}$. The restriction of $F$ to $L_{0}$ is denoted by $F_{0}$. We know from Lemma 4.1 (and Remark 4.2) that there exists a lattice isomorphism $\varphi: L^{\prime} \rightarrow L_{0}$. After replacing $F$ by $F^{\text {mir }}$ if necessary, we 
obtain from Lemma 4.7 that $\varphi:\left(L^{\prime}, D^{\prime}\right) \rightarrow\left(L_{0}, F_{0}\right)$ is a directed diagram isomorphism. Since 3.1(iii) is based on concepts preserved by this sort of isomorphisms, $\varphi\left(\mathcal{H}^{\prime}\right)=\left\{\varphi(I): I \in \mathcal{H}^{\prime}\right\}$ is a nontrivial patchwork system for $F_{0}$. Hence 6.1 (viii) yields a nontrivial patchwork system for $F$, proving that $L$ is patchwork-reducible. This proves (i).

To prove the "if" part of (v), we assume that $L$ is HDc-decomposable; we have to show that so is $L^{\prime}$. By the assumption, there are a proper ideal $I$ and a proper filter $F$ of $L$ such that $L=I \cup F$ and $C:=I \cap F$ is a chain. Let $I^{\prime}:=I \cap L^{\prime}, F^{\prime}:=F \cap L^{\prime}$ and $C^{\prime}:=C \cap L^{\prime}=I^{\prime} \cap F^{\prime}$. Clearly, $L^{\prime}=I^{\prime} \cup F^{\prime}$. Assume for a contradiction that $C^{\prime}=\emptyset$. Then $C$ contains an eye $e$ since $C \neq \emptyset$. Using that $C^{\prime}$ is empty and $\left\{e^{-}, e^{+}\right\} \subseteq L^{\prime}$, we infer that $\left\{e^{-}, e^{+}\right\} \cap C=\emptyset$. It follows from $e^{-}<e \in I$ and $e^{-} \notin C$ that $e^{-} \in I \backslash F$. Dually, we obtain that $e^{+} \in F \backslash I$. Using $L=I \cup F$, we have that $a=a_{e} \in I$ or $a \in F$. However, $a \in I$ gives that $e^{+}=e \vee a \in I$, contradicting $e^{+} \in F \backslash I$, while $a \in F$ gives that $e^{-}=e \wedge a \in F$, contradicting $e^{-} \in I \backslash F$. This contradiction yields that $C^{\prime}$ is nonempty, indeed. So $C^{\prime}$ is a chain since $C^{\prime} \subseteq C$. Since $0_{L}$ and $1_{L}$ are not eyes, they belong to $L^{\prime}$, and $1_{L^{\prime}}=1_{L}$ and $0_{L^{\prime}}=0_{L}$. Hence if $1_{L^{\prime}}$ belonged to $I^{\prime}$, then $1_{L} \in I$ would contradict $I \neq L$. Therefore, $1_{L^{\prime}} \notin I^{\prime}$ shows that $I^{\prime}$ is a proper ideal of $L^{\prime}$. Working with $0_{L^{\prime}}=0_{L}$ dually, we obtain that $F^{\prime}$ is a proper filter of $L^{\prime}$. Thus, $L^{\prime}$ is HDc-decomposable, indeed. This proves the "if" part of $(\mathrm{v})$.

To prove the "only if" part of (v), we next assume that $L^{\prime}$ is HDc-decomposable, and we have to show that so is $L$. By the assumption, there are $u, v \in L^{\prime}$ such that $L^{\prime}=[0, v]_{L^{\prime}} \cup[u, 1]_{L^{\prime}}, 0<u \leq v<1$, and $C^{\prime}:=[u, v]_{L^{\prime}}=[0, v]_{L^{\prime}} \cap[u, 1]_{L^{\prime}}$, understood in $L^{\prime}$, is a chain. Define $I:=[0, v]_{L}$ and $F:=[u, 1]_{L}$, understood in $L$. Then $I$ and $F$ are proper subsets of $L$ since $u \neq 0$ and $v \neq 1$. Assume for a contradiction that $C:=[u, v]_{L}=I \cap F$ contains an eye. Then $e \in[u, v]_{L} \backslash\{u, v\}$ since $e \in L \backslash L^{\prime}$. Hence $e^{-}, e^{+} \in[u, v]_{L}$ implies that the 4-cell $S$ is included in the chain $C^{\prime}=[u, v]_{L^{\prime}}$, a contradiction. Thus, we conclude that $C=C^{\prime}$, whence $C$ is a chain in $L$. Assume for a next contradiction that $L \neq I \cup F$. Then there is an eye $e$ such that $e \notin I=[0, v]_{L}$ and $e \notin F=[u, 1]_{L}$. Hence $e^{+} \notin[0, v]_{L}$ and $e^{-} \notin[u, 1]_{L}$. Using $e^{-}=a \wedge b$ and $e^{+}=a \vee b$, we conclude that $\{a, b\} \nsubseteq[0, v]_{L}$ and $\{a, b\} \nsubseteq[u, 1]_{L}$. In fact, it is more reasonable to write $\{a, b\} \nsubseteq[0, v]_{L^{\prime}}$ and $\{a, b\} \nsubseteq[u, 1]_{L^{\prime}}$ since $a, b \in L^{\prime}$. On the other hand, $\{a, b\} \subseteq L^{\prime}=[0, v]_{L^{\prime}} \cup[u, 1]_{L^{\prime}}$. Therefore, we have


$e^{-}<b$ and $b \in[u, 1]_{L^{\prime}}$ that $e^{-}<e^{-} \vee u \leq b$. This together with $e^{-} \prec b$ implies that $e^{-} \vee u=b$. We know that $u$ belongs to $[0, v]_{L^{\prime}}$. Since $e^{-} \leq a \in[0, v]_{L^{\prime}}$, we have that $e^{-}$also belongs to $[0, v]_{L^{\prime}}$. Therefore, $b=e^{-} \vee u \in[0, v]_{L^{\prime}}$, which is a contradiction. Thus, $L=I \cup F$, and $L$ is HDc-decomposable. This proves (v).

\section{Getting RiD OF Diagrams}

The fact that many of our concepts depends (at least formally) on the diagram chosen causes a lot of inconvenience. The aim of this section is to get rid of this difficulty by proving Proposition 3.2. The following lemma is not surprising.

Lemma 7.1. Let $I$ be a rectangular interval of a planar semimodular lattice $L$, and let $D \in \operatorname{Diag}(L)$. Assume that $x \in I \backslash \mathcal{B}_{\text {right }}^{D}(I)$. Then the right neighbor of $x$ (in $L$, with respect to $D$ ) exists, and it belongs to $I$.

Proof. Let $C_{0}$ and $C_{1}$ be maximal chains in $\downarrow 0_{I}$ and $\uparrow 1_{I}$, respectively. Clearly, there are a unique $s \in \mathcal{B}_{\text {left }}^{D}(I)$ and a unique $t \in \mathcal{B}_{\text {right }}^{D}(I)$ such that $h(s)=h(t)=h(x)$. 
If we had that $x \in \mathcal{B}_{\text {right }}^{D}(L)$, then the left-right dual of (5.3), applied to $I$ and also to $L$, would imply that $x$ and $t$ mutually on the right of each other, whence $(5.2)$ would yield that $x=t \in \mathcal{B}_{\text {right }}^{D}(I)$, a contradiction. Hence $x \notin \mathcal{B}_{\text {right }}^{D}(L)$. Therefore, in virtue of (5.4), the (unique) right neighbor $y$ of $x$ makes sense. Moreover, the left-right dual of (5.3) together with $x \notin \mathcal{B}_{\text {right }}^{D}(I)$ implies that $t$ is strictly on the right of $x$. Consequently, $y$ is on the left of $t$, whence $y$ is on the left of the maximal chain $C_{0} \cup \mathcal{B}_{\text {right }}^{D}(I) \cup C_{1}$. On the other hand, $s$ is on the left of $x$ by (5.3), which yields that $s$ is on the left of $y$. This gives that $y$ is on the right of $C_{0} \cup \mathcal{B}_{\text {left }}^{D}(I) \cup C_{1}$. Finally, $y \in I$ follows in virtue of (5.6).

Proof of Proposition 3.2. In order to show that (i) $\Rightarrow$ (ii), we assume (i). Let $D \in \operatorname{Diag}(L)$ be a diagram witnessing that 3.1(iii) holds. Consider a pair $(I, J) \in$ $\mathcal{E}(\mathcal{H})$. By the assumptions, $I \cap J$ is a chain and, say, $I \cap J \subseteq \mathcal{B}_{\text {north }}^{D}(I) \cap \mathcal{B}_{\text {south }}^{D}(J)$. Let $F \in \operatorname{Diag}(L)$ be another diagram. We already know from Lemma 5.5 that $I \cap J \subseteq \mathcal{B}^{F}(I) \cap \mathcal{B}^{F}(J)$. If we had an element $x \in I \cap J$ such that $x \in \mathcal{B}^{F}(I) \backslash \mathcal{B}_{\text {north }}^{F}(I)$, then $x$ would have at least two covers within $I$ by $(2.14)$, applied to $F$, but this would contradict (2.13), applied to $D$. Hence $I \cap J \subseteq \mathcal{B}_{\text {north }}^{F}(I)$. Similarly, if we had an element $x \in I \cap J$ such that $x \in \mathcal{B}^{F}(J) \backslash \mathcal{B}_{\text {south }}^{F}(J)$, then $x$ would have at least two lower covers within $J$ by (2.15), applied to $F$, but this would contradict (2.13), applied to $D$. Hence $I \cap J \subseteq \mathcal{B}_{\text {south }}^{F}(J)$. Thus $I \cap J \subseteq \mathcal{B}_{\text {north }}^{F}(I) \cap \mathcal{B}_{\text {south }}^{F}(J)$, which means that (ii) holds.

Next, to show that (ii) $\Rightarrow$ (iv), we assume (ii). Let $D \in \operatorname{Diag}(L)$. By the $I$ - $J$ symmetry, we can assume that $I \cap J \subseteq \mathcal{B}_{\text {north }}^{D}(I) \cap \mathcal{B}_{\text {south }}^{D}(J)$. Assume for a contradiction that $I \cap J \nsubseteq \mathcal{B}_{\mathrm{ne}}^{D}(I)$ and $I \cap J \nsubseteq \mathcal{B}_{\mathrm{nw}}^{D}(I)$. Then there are $x, y \in I \cap J$ such that $x \in \mathcal{B}_{\text {nw }}^{D}(I) \backslash \mathcal{B}_{\text {ne }}^{D}(I)$ and $y \in \mathcal{B}_{\text {ne }}^{D}(I) \backslash \mathcal{B}_{\mathrm{nw}}^{D}(I)$. Since $I \cap J$ is a chain, we can assume by left-right symmetry that $x \leq y$. Using (2.12) and Lemma 4.9(ii), we obtain that $1_{I}=w_{D}^{\ell}(I) \vee w_{D}^{r}(I) \leq x \vee y=y \leq 1_{I}$, which gives that $y=1_{I} \in \mathcal{B}_{\mathrm{nw}}^{D}(I)$, a contradiction. This shows that

$$
I \cap J \subseteq \mathcal{B}_{\mathrm{nw}}^{D}(I) \quad \text { or } \quad I \cap J \subseteq \mathcal{B}_{\mathrm{ne}}^{D}(I)
$$

The dual argument yields that

$$
I \cap J \subseteq \mathcal{B}_{\mathrm{sw}}^{D}(J) \quad \text { or } \quad I \cap J \subseteq \mathcal{B}_{\mathrm{se}}^{D}(J) .
$$

We can assume that the disjunction "or" is an exclusive disjunction both in (7.1) and (7.2) since otherwise the desired 3.2(iiib) for $D$ trivially holds. Hence, by the left-right symmetry and keeping the targeted 3.2(iiib) in mind, we can assume for a contradiction that

$$
I \cap J \subseteq \mathcal{B}_{\mathrm{nw}}^{D}(I), I \cap J \nsubseteq \mathcal{B}_{\mathrm{ne}}^{D}(I), I \cap J \subseteq \mathcal{B}_{\mathrm{sw}}^{D}(J) \text {, and } I \cap J \nsubseteq \mathcal{B}_{\mathrm{se}}^{D}(J)
$$

Firstly, we assume that there is a $u \in(I \cap J) \backslash\left\{1_{I}, 0_{J}\right\}$. Then $u \notin \mathcal{B}_{\text {right }}^{D}(I)$ since $\mathcal{B}_{\text {nw }}^{D}(I) \cap \mathcal{B}_{\text {right }}^{D}(I)=\left\{1_{I}\right\}$. Similarly, $u \notin \mathcal{B}_{\text {right }}^{D}(J)$ since $\mathcal{B}_{\text {sw }}^{D}(J) \cap \mathcal{B}_{\text {right }}^{D}(J)=\left\{0_{J}\right\}$. Hence, by Lemma 7.1, the right neighbor $v$ of $u$ with respect to $D$ exists, and it belongs to $I \cap J$. However, then $u \| v$ and $u, v \in I \cap J$ is a contradiction since $I \cap J$ is a chain.

Secondly, we assume that there is no such $u$. By (7.3), we can select $x, y \in I \cap J$ such that $x \in \mathcal{B}_{\text {nw }}^{D}(I) \backslash \mathcal{B}_{\text {ne }}^{D}(I)$ and $y \in \mathcal{B}_{\text {sw }}^{D}(J) \backslash \mathcal{B}_{\text {se }}^{D}(J)$. Notice that $x \notin \mathcal{B}_{\text {right }}^{D}(I)$ since $I \cap J \subseteq \mathcal{B}_{\text {north }}^{D}(I)$, and $y \notin \mathcal{B}_{\text {right }}^{D}(J)$ since $I \cap J \subseteq \mathcal{B}_{\text {south }}^{D}(J)$. If we had $x \geq y$, then $u:=x$ (or $u:=y$ ) would lead to the previous case. Hence we assume that $x<y$. If we had $x \neq 0_{J}$ or $y \neq 1_{I}$, then $u:=x$ or $u:=y$ would again lead to the previous case. Hence $x=0_{J}$ and $y=1_{I}$. We can also assume that $0_{J}=x \prec y=1_{I}$ since 
otherwise, using the convexity of $I \cap J$, we could choose a $u \in I \cap J \cap[x, y] \backslash\{x, y\}$, which would lead to the previous case again. Let $z$ be the unique atom of $J$ that belongs to $\mathcal{B}_{\text {right }}^{D}(J)$. Similarly, let $t$ be the unique coatom of $I$ that belongs to $\mathcal{B}_{\text {right }}^{D}(I)$.

Extend $\mathcal{B}_{\text {right }}^{D}(I) \cup\left\{s \in \mathcal{B}_{\text {left }}^{D}(J): s \geq y=1_{I}\right\}$ to a maximal chain $C$ of $L$. Since $h\left(0_{J}\right)=h\left(1_{I}\right)-1=h(t) \in \mathcal{B}_{\text {right }}^{D}(I) \subseteq C$ and $0_{J}=x \notin \mathcal{B}_{\text {right }}^{D}(I)$, we obtain from the left-right dual of (5.3) that $0_{J}$ is strictly on the left of $t$. Hence (5.1) yields that $0_{J}$ is strictly on the left of $C$. Similarly, $1_{I}=y \notin \mathcal{B}_{\text {right }}^{D}(J)$ together with the left-right dual of (5.3) gives that $z$ is strictly on the right of $1_{I}=y \in C$, whence (5.1) yields that $z$ is strictly on the right of $C$. However, then $0_{J} \prec z$ contradicts (2.5). Thus, (7.3) leads to a contradiction, proving (ii) $\Rightarrow$ (iv).

The implication (iii) $\Rightarrow$ (i) is evident. So is (iv) $\Rightarrow$ (iii) since $L$ is planar.

\section{Patch lattices}

We are not in the position of proving Theorem 3.4 yet. However, some of its parts will be needed in the next sections. Therefore, now we prove a part of it.

Lemma 8.1. (ii) $\Leftrightarrow$ (iii) $\Leftrightarrow$ (iv) $\Leftrightarrow$ (v) $\Leftrightarrow$ (vi) of Theorem 3.4 hold.

Proof. (ii) $\Rightarrow$ (iii) is obvious.

By Lemma 6.1, it suffices to prove the implication (iii) $\Rightarrow$ (iv) only for slim semimodular lattices. Hence assume that $L$ is a slim semimodular lattice and (iii) holds. Let $D \in \operatorname{Diag}(L)$. We know from (2.4) that there is a double irreducible element in $\mathcal{B}_{\text {left }}^{D}(L) \backslash\{0,1\}$. In fact, there is a smallest one since $\mathcal{B}_{\text {left }}^{D}(L)$ is a chain; we denote it by $a$. Let $b_{0}$ denote the smallest element of $\mathcal{B}_{\text {right }}^{D}(L) \backslash \downarrow a$, and let $b_{0}^{-}$ be the unique lower cover of $b_{0}$ that belongs to $\mathcal{B}_{\text {right }}^{D}(L)$. Let $c:=a \vee b_{0}$. From semimodularity and $a=a \vee b_{0}^{-}$we obtain that $a \prec c$. Since $\mathcal{B}_{\text {left }}^{D}(L)$ is a chain and $a \in \mathcal{B}_{\text {left }}^{D}(L)$ has exactly one cover in $L$, we conclude that $c \in \mathcal{B}_{\text {left }}^{D}(L)$. Let $b$ be the largest element of $\mathcal{B}_{\text {right }}^{D}(L) \cap \downarrow c$. Then $c=a \vee b$ since $b_{0} \leq b \leq c$ and $c=a \vee b_{0}$.

Assume that $z_{1}, z_{2} \in \downarrow c \cap \uparrow b=[b, c]$. By (2.10), there are $x_{i} \in \mathcal{B}_{\text {left }}^{D}(L)$ and $y_{i} \in \mathcal{B}_{\text {right }}^{D}(L)$ such that $z_{i}=x_{i} \vee y_{i}$, for $i \in\{1,2\}$. By the definition of $b$ and $z_{i} \leq c$, we know that $y_{i} \leq b$. Hence $z_{i}=z_{i} \vee b=x_{i} \vee b$, for $i \in\{1,2\}$. Since $x_{1}, x_{2} \in \mathcal{B}_{\text {left }}^{D}(L)$ are comparable, so are $z_{1}$ and $z_{2}$. This together with $b \leq c$ shows that $\downarrow c \cap \uparrow b$ is a chain. Next, consider an arbitrary $z \in L$; we want to show that $z \in \downarrow c \cup \uparrow b$. By (2.10), $z=x \vee y$ for some $x \in \mathcal{B}_{\text {left }}^{D}(L)$ and $y \in \mathcal{B}_{\text {right }}^{D}(L)$. We can assume that $y<b$ since otherwise $z \in \uparrow b$. Then we can assume that $c<x$ since otherwise $z=x \vee y \leq c \vee b=c$ would mean that $z \in \downarrow c$. Therefore, $b \leq c<x \leq x \vee y=z$, that is, $z \in \uparrow b$. This shows that $L=\downarrow c \cup \uparrow b$.

Thus, by (iii), either $\downarrow c=L$ or $\uparrow b=L$. But $b_{0}^{-}<b_{0} \leq b$ excludes the latter, so $\downarrow c=L$, which means that $c=1$. This shows that the smallest (and therefore every) doubly irreducible element on the left boundary is a coatom. In particular, there is exactly one left weak corner with respect to $D$; it is a coatom and it will be denoted by $w_{D}^{\ell}$. Similarly, there is exactly one right weak corner $w_{D}^{r}$. Since $L$ is glued sum indecomposable, $\mathcal{B}_{\text {left }}^{D}(L) \cap \mathcal{B}_{\text {right }}^{D}(L)=\{0,1\}$. This yields that $w_{D}^{\ell} \neq w_{D}^{r}$. Hence $w_{D}^{\ell} \vee w_{D}^{r}=1$ since they are coatoms. If $w_{D}^{\ell} \wedge w_{D}^{r}=0$, then (iv) is clear.

Assume for a contradiction that $\downarrow w_{D}^{\ell}$ is not a chain. By Lemma 2.3, $\downarrow w_{D}^{\ell}$ has a glued some indecomposable component $A$. Obviously, $\mathcal{B}_{\text {left }}^{D}(A) \cap \mathcal{B}_{\text {right }}^{D}(A)=$ $\left\{0_{A}, 1_{A}\right\}$. Hence (2.4) yields an element $s \in \mathcal{B}_{\text {left }}^{D}(A) \backslash \mathcal{B}_{\text {right }}^{D}(A)$ such that $s$ is doubly irreducible within $A$. It is obvious by Lemma 2.3 that $\mathcal{B}_{\text {right }}^{D}(A) \subseteq \mathcal{B}_{\text {right }}^{D}\left(\downarrow w_{D}^{\ell}\right)$ and, 
taking (5.5) into account, $\mathcal{B}_{\text {left }}^{D}(A) \subseteq \mathcal{B}_{\text {left }}^{D}\left(\downarrow w_{D}^{\ell}\right) \subseteq \mathcal{B}_{\text {left }}^{D}(L)$. Hence we conclude that $s \in \mathcal{B}_{\text {left }}^{D}(L) \backslash \mathcal{B}_{\text {right }}^{D}\left(\downarrow w_{D}^{\ell}\right)$. Moreover, $s$ is doubly irreducible also within $\downarrow w_{D}^{\ell}$. Let $s^{+}$ denote the unique cover of $s$ in $\mathcal{B}_{\text {left }}^{D}\left(\downarrow w_{D}^{\ell}\right) \subseteq \mathcal{B}_{\text {left }}^{D}(L)$. Evidently, $s$ is join-irreducible not only in $\downarrow w_{D}^{\ell}$ but also in $L$. Since $w_{D}^{\ell}$ is the only doubly irreducibly element (that is, a weak left corner) on the left boundary of $L$ and $s<1_{A} \leq w_{D}^{\ell}$, we conclude that $s$ is meet-reducible in $L$. Therefore, $s$ has a cover $s^{\prime} \in L \backslash \downarrow w_{D}^{\ell}$. Notice that $s^{\prime} \neq s^{+}$. Hence (5.3) yields that $s^{\prime}$ is strictly on the right of $s^{+}$, and we obtain from (5.1) that $s^{\prime}$ is strictly on the right of the maximal chain $\mathcal{B}_{\text {left }}^{D}(L)=\mathcal{B}_{\text {left }}^{D}\left(\downarrow w_{D}^{\ell}\right) \cup\{1\}$. If $s^{\prime}$ was on the left of the maximal chain $C:=\mathcal{B}_{\text {right }}\left(\downarrow w_{D}^{\ell}\right) \cup\{1\}$, then (5.6) (with 0 and $w_{D}^{\ell}$ acting as $u$ and $v$, respectively) would imply that $s^{\prime} \in \downarrow w_{D}^{\ell}$. Therefore

$s^{\prime}$ is strictly on the right of $C$.

Let $t \in \mathcal{B}_{\text {right }}^{D}(A) \subseteq \mathcal{B}_{\text {right }}^{D}\left(\downarrow w_{D}^{\ell}\right) \subseteq C$ be the unique element with $h(t)=h(s)$. Then $s \neq t$ since $s \notin \mathcal{B}_{\text {right }}^{D}(A)$. It follows from (5.3) that $s$ is strictly on the left of $t$. Hence (5.1) gives that $s$ is strictly on the left of $C$. However, this fact together with (8.1) and $s \prec s^{\prime}$ contradicts (2.5), proving that $\downarrow w_{D}^{\ell}$ is a chain.

Therefore, $\downarrow w_{D}^{\ell} \subseteq \mathcal{B}_{\text {left }}^{D}(L)$ and, similarly, $\downarrow w_{D}^{r} \subseteq \mathcal{B}_{\text {right }}^{D}(L)$. Combining this with the glued sum indecomposability of $L$, we conclude that $w_{D}^{\ell} \wedge w_{D}^{r} \in \mathcal{B}_{\text {left }}^{D}(L) \cap$ $\mathcal{B}_{\text {right }}^{D}(L)=\{0,1\}$. This gives the desired $w_{D}^{\ell} \wedge w_{D}^{r}=0$. Thus, (iv) holds, proving the implication (iii) $\Rightarrow$ (iv).

The implication (iv) $\Rightarrow(\mathrm{v})$ is evident.

Assume (v). Then $L$ has two coatoms whose meet is 0 , whence (vi) follows easily from Lemma 5.4. This proves that $(\mathrm{v}) \Rightarrow$ (vi).

To show (vi) $\Rightarrow$ (ii), take a fixed planar diagram of $L$. Let $a$ and $b$ be the leftmost and the rightmost coatoms of $L$, respectively. Assume that $I$ is an ideal and $F$ is a filter of $L$ such that $L=I \cup F$ and $I \cap F \neq \emptyset$. We have to show that $L \in\{I, F\}$. If $a, b \in F$, then $F=L$ since $0=a \wedge b \in F$. If $a, b \in I$, then $I=L$ since $1=a \vee b \in I$. Therefore, since $\{a, b\} \subseteq L=I \cup F$, we can assume that, say, $a \in I$ and $b \in F$. Consider the smallest element of $I \cap F$. Clearly, it is $0_{F}$. If $0_{F}=0$, then $F=L$. Hence we can assume that $0<0_{F}$. Since $0_{F} \leq a$ would lead to the contradiction $0<0_{F} \leq a \wedge b=0$, we conclude that $0_{F} \not \leq a$. Hence $1=a \vee 0_{F} \in I$, implying that $I=L$. Thus, (vi) $\Rightarrow$ (ii).

\section{Some PROPERTIES OF PATCH INTERVALS}

The lemmas of this section formulate some properties of patch intervals, also called patches, of $L$. Eventually, these properties will be easy consequences of Theorem 3.6. However, we have to prove them now since they will be used in the proof of Theorem 3.6.

Lemma 9.1. Let $I$ and $J$ be patches of a slim semimodular lattice $L$ such that $0_{J} \in I \backslash \mathcal{B}_{\text {north }}(I)=I \backslash\left\{1_{I}, w_{I}^{\ell}, w_{I}^{r}\right\}$. Then $I \subseteq J$ or $J \subseteq I$. (By Lemma 4.9, the choice of $D \in \operatorname{Diag}(L)$ is irrelevant.)

Proof. Assume that $J \nsubseteq I$. Then $\left\{w_{J}^{\ell}, w_{J}^{r}\right\} \nsubseteq I$ since otherwise $1_{J}=w_{J}^{\ell} \vee w_{J}^{r} \in I$ and the convexity of $I$ would imply that $J \subseteq I$. Let, say, $w_{J}^{\ell} \notin I$. Applying Lemma 5.1 to $0_{J}<w_{J}^{\ell}$, we obtain an element $x \in \mathcal{B}_{\text {north }}(I)=\mathcal{B}_{\text {north }}^{D}(I)$ such that $0_{J} \leq x \leq w_{J}^{\ell}$. In fact, we have that $0_{J}<x<w_{J}^{\ell}$ by the assumptions. There are four cases to consider. 
Case 1. Assume that $x=w_{I}^{r}$. Since $0_{J}<x=w_{I}^{r}$ and $\left[0_{I}, w_{I}^{r}\right]=\mathcal{B}_{\mathrm{se}}(I)$ is a chain by $(2.11)$ and $(2.12), 0_{J}$ has a unique cover $y_{1}$ in $\mathcal{B}_{\text {se }}(I) \subseteq \mathcal{B}_{\text {right }}(I)$. By (2.14), $0_{J}$ has another cover $y_{0} \in I$, which is strictly on the left of $y_{1}$. Since $y_{1} \in\left[0_{J}, w_{I}^{r}\right] \subset\left[0_{J}, w_{J}^{\ell}\right] \subseteq \mathcal{B}_{\text {left }}(J),(2.14)$ yields that $0_{J}$ has a cover $y_{2} \in \mathcal{B}_{\text {right }}(J)$, which is strictly on the right of $y_{1}$. Their position shows that $y_{0}, y_{1}$ and $y_{2}$ are three distinct covers of $0 J$. Thus, the present case is excluded by (2.1).

Case 2. Assume that $x=w_{I}^{\ell}$ and $w_{J}^{r} \in I$. Then $w_{I}^{\ell}<w_{J}^{\ell}$. This together with $w_{J}^{\ell} \nsupseteq w_{J}^{r}$ give that $w_{I}^{\ell} \geq w_{J}^{r}$. So $w_{I}^{\ell}<w_{I}^{\ell} \vee w_{J}^{r} \in I$ yields that $w_{I}^{\ell} \vee w_{J}^{r}=1_{I}$. Since $J \nsubseteq I$, we know that $1_{J} \neq 1_{I}$. But $1_{I}=w_{I}^{\ell} \vee w_{J}^{r} \leq w_{J}^{\ell} \vee w_{J}^{r}=1_{J}$, so $w_{J}^{r} \leq 1_{I}<1_{J}$. Combining this with $w_{J}^{r} \prec 1_{J}$ we obtain that $w_{J}^{r}=1_{I}$. Hence $w_{I}^{\ell} \leq w_{J}^{\ell} \wedge 1_{I}=w_{J}^{\ell} \wedge w_{J}^{r}=0_{J}$ and $0_{J} \leq x=w_{I}^{\ell}$ give that $0_{J}=w_{I}^{\ell}$, contradicting the assumptions of the lemma. Thus, this case is excluded again.

Case 3. Assume that $x=w_{I}^{\ell}$ and $w_{J}^{r} \notin I$. Again, we know that $w_{I}^{\ell}<w_{J}^{\ell}$. Applying Lemma 5.1 to $0_{J}<w_{J}^{r}$, we obtain a $y \in \mathcal{B}_{\text {north }}(I)=\left\{w_{I}^{\ell}, w_{I}^{r}, 1_{I}\right\}$ such that $0_{J} \leq$ $y \leq w_{J}^{r}$. If we had that $y \in\left\{w_{I}^{\ell}, 1_{I}\right\}$, then $w_{I}^{\ell} \leq y<w_{J}^{r}$ together with $w_{I}^{\ell}<w_{J}^{\ell}$ would give that $w_{I}^{\ell} \leq w_{J}^{\ell} \wedge w_{J}^{r}=0_{J} \in I$, implying $0_{J} \in\left\{w_{I}^{\ell}, 1_{I}\right\}$, a contradiction. Hence $y=w_{I}^{r}$, and we have that $w_{I}^{r} \leq w_{J}^{r}$. By the definition of $x$ and $y$, we know that $w_{I}^{\ell} \in\left[0_{J}, w_{J}^{\ell}\right]$ and $w_{I}^{r} \in\left[0_{J}, w_{J}^{r}\right]$. Hence $0_{J} \leq w_{I}^{\ell} \wedge w_{I}^{r} \leq w_{J}^{\ell} \wedge w_{J}^{r}=0_{J}$, that is, $0_{J}=w_{I}^{\ell} \wedge w_{I}^{r}=0_{I}$. This and $1_{J}=w_{J}^{\ell} \vee w_{J}^{r} \geq w_{I}^{\ell} \vee w_{I}^{r}=1_{I}$ yields that $I \subseteq J$, as desired.

Case 4. Assume that $x=1_{I}$. Applying Lemma 5.1 to $0_{J}<w_{J}^{r}$ again, we obtain a $y \in \mathcal{B}_{\text {north }}(I)=\left\{w_{I}^{\ell}, w_{I}^{r}, 1_{I}\right\}$ such that $0_{J} \leq y \leq w_{J}^{r}$. The possibility $y \in\left\{w_{I}^{\ell}, w_{I}^{r}\right\}$ belongs, apart from notation and left-right symmetry, to the scope of the previous three cases. Hence we can assume that $y=1_{I}$. However, then $0_{J} \leq x \wedge y \leq$ $w_{J}^{\ell} \wedge w_{J}^{r}=0_{J}$ implies that $0_{J}=x \wedge y=1_{I} \wedge 1_{I}=1_{I}$, contradicting the assumptions of the lemma. So this case is excluded.

Lemma 9.2. Let $I$ and $J$ be maximal patches of a planar semimodular lattice $L$. If they have the same top, then they coincide. Moreover, $0_{I}$ is the intersection of all lower covers of $1_{I}$.

Proof. Let us fix a planar diagram $D$ of $L$, and keep Lemma 4.9(ii) in mind. Alternatively, no matter how $D$ is fixed since the concept of a (maximal) patch interval does not depend on $D$; this fact is due to (ii) or (iii) of Lemma 8.1, which clearly do not depend on $D$. With respect to $D$, let $a$ and $b$ be the leftmost and the rightmost lower cover of $1_{I}=1_{J}$, respectively, and let $u:=a \wedge b$. Then $a$ and $b$ are the leftmost coatom and the rightmost coatom of $K:=\left[u, 1_{I}\right]$. By the (iv) $\Leftrightarrow$ (v) part of Lemma 8.1, we conclude that $K \in \mathcal{P}(L)$. Since $w_{I}^{\ell}, w_{I}^{r}, w_{J}^{\ell}, w_{J}^{r} \in K$ by Lemma 5.4, $0_{I}=w_{I}^{\ell} \wedge w_{I}^{r}$ and $0_{J}$ also belong to $K$. Hence $I, J \subseteq K$. Therefore, $I, J \in \mathcal{P}_{\max }(L)$ yields that $I=K=J$. We have also obtained that $0_{I}=0_{K}=a \wedge b$. In virtue of Lemma 5.4, this proves the second part.

Lemma 9.3. Let $I$ and $J$ be maximal patches of a slim semimodular lattice $L$. Then either $I=J$, or $I$ and $J$ are disjoint, or $I \cap J$ is a chain.

Proof. Let $D \in \operatorname{Diag}(L)$ be fixed. Let $a, b \in I \cap J$ such that $a \| b$; we have to show that $I=J$. By Lemma 9.2 , this is clear if $1_{I}=1_{J}$. Assume, by way of contradiction, that $1_{I} \neq 1_{J}$. Then, say, $1_{I} \nsucceq 1_{J}$. Lemma 5.1 , applied to $I$ and $a, b \leq 1_{J}$, yields elements $a^{\prime}, b^{\prime} \in\left\{w_{I}^{\ell}, w_{I}^{r}, 1_{I}\right\}$ such that $a \leq a^{\prime}<1_{J}$ and $b \leq b^{\prime}<1_{J}$. 
If $1_{I} \in\left\{a^{\prime}, b^{\prime}\right\}$, then $1_{I} \leq 1_{J}$. Otherwise, if we had $a^{\prime}=b^{\prime} \in\left\{w_{I}^{\ell}, w_{I}^{r}\right\}$, then $a$ and $b$ would belong to the same chain (in $I$ ) by (2.11) and (2.12), which would contradict $a \| b$. Hence $\left\{a^{\prime}, b^{\prime}\right\}=\left\{w_{I}^{\ell}, w_{I}^{r}\right\}$, which gives that $1_{I}=w_{I}^{\ell} \vee w_{I}^{r}=a^{\prime} \vee b^{\prime} \leq 1_{J}$. Hence, in all cases, $1_{I} \leq 1_{J}$. So $1_{I}<1_{J}$ since they are distinct. Therefore, the convexity of $J, a \in J$ and $a \leq 1_{I}<1_{J}$ yield that $1_{I} \in J \backslash\left\{1_{J}\right\}$.

Assume first that $1_{I} \in \mathcal{B}(J)$; then $1_{I} \in \mathcal{B}(J) \backslash\left\{1_{J}\right\}=\mathcal{B}_{\text {south }}(J)$. Let, say, $1_{I} \in \mathcal{B}_{\mathrm{sw}}(J)$. Then $a \| b$ belong to the same chain $\mathcal{B}_{\mathrm{sw}}(J)$ of $J$ by by $(2.11)$ and (2.12), a contradiction. Hence $1_{I}$ is in the interior of $J$, whence its lower covers, $w_{I}^{\ell}$ and $w_{I}^{r}$, belong to $J$ by (2.6) and (2.8). Consequently, $0_{I}=w_{I}^{\ell} \wedge w_{I}^{r} \in J$. Hence $0_{J} \leq 0_{I}<1_{I}<1_{J} \in J$ yields that $I \subset J$, contradicting $I, J \in \mathcal{P}_{\max }(L)$.

Lemma 9.4. Let $L$ be a slim semimodular lattice with a fixed $D \in \operatorname{Diag}(L)$, and let $I, J \in \mathcal{P}_{\max }(L)$ such that $|I \cap J|=1$. Then, up to $I$-J and left-right symmetries, either $I \cap J=\left\{w_{D}^{r}(I)\right\}=\left\{w_{D}^{\ell}(J)\right\}$, or $I \cap J=\left\{1_{I}\right\}=\left\{0_{J}\right\}$.

The direct square $3^{2}$ of the three-element chain shows that both cases can occur.

Proof of Lemma 9.4. Let $x$ denote the unique element of $I \cap J$. There are several cases to consider.

Case 1. Assume that $x \in\left\{0_{I}, 1_{I}, 0_{J}, 1_{J}\right\}$. Firstly, let $x \in\left\{1_{I}, 1_{J}\right\}$, say, $x=1_{I}$. Since $I$ contains all lower covers of $x$ by Lemma 9.2 but none of these lower covers are in $J$ since $|I \cap J|=1$, we conclude that $x=0_{J}$, as desired. Secondly, let $x \in\left\{0_{I}, 0_{J}\right\}$, say, $x=0_{I}$. By (2.1) and the definition of a patch lattice, $x$ has exactly two covers in $L$, and both covers of $x$ belong to $I$. Since none of these covers can belong to $J$ by $|I \cap J|=1$, we obtain that $x=1_{J}$, as desired.

Case 2. Assume for a contradiction that $x \in \operatorname{int}(I) \cup \operatorname{int}(J)$. Say, $x \in \operatorname{int}(J)$. Then, by (2.6) and (2.8), all upper covers of $x$ belong to $J$. But none of them can belong to the singleton set $I \cap J$, whence we obtain $x=1_{I}$. By the previous case, this implies that $0_{J}=x \in \operatorname{int}(J)$, a contradiction.

Case 3. Next, assume for a contradiction that $x \in \mathcal{B}_{\text {south }}(J) \backslash\left\{0_{J}, w_{D}^{\ell}(J), w_{D}^{r}(J)\right\}$, or the same holds for $I$. Then $x$ has exactly two covers , $x_{1}$ and $x_{2}$, in $J$ by $(2.1)$ and (2.14). Since $\left\{x_{1}, x_{2}\right\} \cap I=\emptyset$ by $|I \cap J|=1,(2.1)$ and the convexity of $I$ imply that $x=1_{I}$. Hence the first case we considered gives that $x=0_{J}$, a contradiction.

Case 4. Assume that $x \in\left\{w_{I}^{\ell}, w_{I}^{r}, w_{J}^{\ell}, w_{J}^{r}\right\}$, where $w_{I}^{\ell}$ stands for $w_{D}^{\ell}(I)$, etc. We can also assume that $x \in\left\{w_{I}^{\ell}, w_{I}^{r}\right\} \cap\left\{w_{J}^{\ell}, w_{J}^{r}\right\}$ since otherwise the situations belongs to the scope of one of the previous cases. To complete the proof, we have to exclude that $x=w_{I}^{\ell}=w_{J}^{\ell}$ or $x=w_{I}^{r}=w_{J}^{r}$. Assume for a contradiction that, say, $x=w_{I}^{\ell}=w_{J}^{\ell}$. We know from Lemma 9.2 that $1_{I}$ is distinct from $1_{J}$. Clearly, both of them cover $x$, whence they are the only covers of $x$ by (2.1). Let, say, $1_{I}$ on the left of $1_{J}$. It follows from semimodularity that $S=\left\{x, 1_{I}, 1_{J}, 1_{I} \vee 1_{J}\right\}$ is a 4-cell with $\mathcal{B}_{\text {left }}(S)=\left\{x, 1_{I}, 1_{I} \vee 1_{J}\right\}$ and $\mathcal{B}_{\text {right }}(S)=\left\{x, 1_{J}, 1_{I} \vee 1_{J}\right\}$. Let $C_{0}$ and $C_{1}$ be maximal chains of $\downarrow x$ and $\uparrow\left(1_{I} \vee 1_{J}\right)$, respectively. Let $W=C_{0} \cup \mathcal{B}_{\text {left }}(S) \cup C_{1}$ and $E=C_{0} \cup \mathcal{B}_{\text {right }}(S) \cup C_{1}$. Since $w_{I}^{r}$ is strictly on the right of $w_{I}^{\ell}=x \in E$ and $h\left(w_{I}^{r}\right)=h\left(w_{I}^{\ell}\right)$, we conclude from the left-right dual of (5.1) that $w_{I}^{r}$ is strictly on the right of $E$. Using $1_{J} \in E$ and $h\left(1_{J}\right)=h(x)+1=h\left(1_{I}\right)$ similarly, we obtain from (5.1) that $1_{I}$ is strictly on the left of $E$. Thus, (2.5) applies to $T$ and $w_{I}^{r} \prec 1_{I}$, and we obtain a contradiction. 
Lemma 9.5. For a slim semimodular lattice $L$ with a fixed $D \in \operatorname{Diag}(L)$, let $I, J \in \mathcal{P}_{\max }(L)$ such that at least one of the following two conditions holds:

(i) $|I \cap J| \geq 3$;

(ii) $J \cap \operatorname{int}^{D}(I)$ is nonempty, or $I \cap \operatorname{int}^{D}(J)$ is nonempty.

Then $I=J$.

Proof. To prove part (i) by way of contradiction, we assume that $I \neq J$ but $|I \cap J| \geq$ 3. We have that $I \| J$ since they are maximal patches. Let $x$ be the least element of $I \cap J$. Since $I \cap J$ is a chain by Lemma 9.3 and $|I \cap J| \geq 3, x \notin \mathcal{B}_{\text {north }}(I)=\mathcal{B}_{\text {north }}^{D}(I)$ and $x \notin \mathcal{B}_{\text {north }}(J)$. It follows from Lemma 9.1 that $x \notin\left\{0_{I}, 0_{J}\right\}$. First we consider the case when $x$ is meet-reducible. Then, by (2.1), $x$ has exactly two covers. Both of these covers belongs to $I$, either since $x \in \mathcal{B}(I) \backslash \mathcal{B}_{\text {north }}(I)$ and (2.14) applies, or since $x \in \operatorname{int}(I)$ and (2.8) together with (2.6) says so. By the same reason, both covers of $x$ belongs to $J$. But this is impossible since $I \cap J$ is a chain. Therefore, $x$ is in $\operatorname{Mi}(L)$, whence also in $\operatorname{Mi}(I) \cap \operatorname{Mi}(J)$. This, $x \notin \mathcal{B}_{\text {north }}(I), x \notin \mathcal{B}_{\text {north }}(J)$ and (2.14) yield that $x \in \operatorname{int}(I) \cap \operatorname{int}(J)$. By (2.6) and (2.8), all lower covers of $x$ are in $I \cap J$. This contradicts the choice of $x$.

To prove part (ii) by way of contradiction, we assume that $x \in \operatorname{int}(I) \cap J$ and $I \neq J$. By Lemma 9.4, $|I \cap J| \neq 1$. Hence $|I \cap J|=2$ by part (i). Since $I \cap J$ is a convex sublattice, it is of the form $\{x, y\}$, where either $x \prec y$, or $y \prec x$.

Assume first that $x \prec y$. If $y$ belonged to $\operatorname{Ji}(I)$, which equals $\mathcal{B}_{\text {south }}(I) \backslash\left\{0_{I}\right\}$ by (2.10) and (2.13), then $x$ would belong to $\mathcal{B}_{\text {south }}(I)$ by $(2.12)$, which would contradict $x \in \operatorname{int}(I)$. Hence $y$ is join-reducible in $I$ and $y \in \operatorname{int}(I) \cup\left\{1_{I}\right\}$. Consequently, $y$ has at least two lower covers in $I$. All lower covers (taken in $L$ ) of $y$ belong to $I$ either since $y=1_{I}$ and Lemma 9.2 applies, or since $y \in \operatorname{int}(I)$ and (2.8) together with (2.6) applies. Since $|I \cap J|=2$, y has only one lower cover (namely, $x$ ) in $J$. That is, $y \in \operatorname{Ji}(J)=\mathcal{B}_{\text {south }}(J) \backslash\left\{0_{J}\right\}$ by $(2.10)$ and (2.13). Hence $x \in \mathcal{B}(J) \backslash \mathcal{B}_{\text {north }}(J)$ by (2.12), and $x$ has exactly two upper covers in $J$ by (2.14) and (2.1). Both of these upper covers belong also to $I$ by (2.6) and (2.8) since $x$ is in the interior of $I$. Therefore, $I \cap J$ has at least three distinct elements, $x$ and its upper covers, which contradicts part (i) of the present lemma.

Secondly, we assume that $y \prec x$. All lower covers of $x$ belong to $I$ by (2.6) and (2.8). Hence $y$ is the only lower cover of $x$ in $J$ since otherwise $|I \cap J| \geq 3$ would contradict part (i) of the present lemma. Consequently, $x \in \operatorname{Ji}(J)=\mathcal{B}_{\text {south }}(J) \backslash\left\{0_{J}\right\}$ by (2.10) and (2.13). Hence $y \in \mathcal{B}(J) \backslash \mathcal{B}_{\text {north }}(J)$ by (2.12). Moreover, $y$ has exactly two upper covers in $J$ (and also in $L$ ) by (2.14) combined with (2.1). These upper covers of $y$ are $x$ and, say, $x^{\prime}$. Since $x \in \operatorname{int}(I)$, either $y \in \operatorname{int}(I)$, or $y \in \mathcal{B}(I) \backslash \mathcal{B}_{\text {north }}(I)$. In both cases, either by (2.6) and (2.8), or by (2.14) combined with (2.1), $x, x^{\prime} \in I$. Hence $x, x^{\prime}$ and $y$ are three distinct elements of $I \cap J$, which contradicts part (i) again.

\section{PRoving the MAIN RESUlts AND Their COROLlaries}

Before accomplishing what is stated in the title of this section, we give the details how Proposition 2.4 is extracted from previous results.

Proof of Proposition 2.4. Part (i) is [7, Lemma 22]. To prove part (ii), observe that if we add forks to a fixed diagram, then the left and the right weak corners, and also the principal filters they determine, do not change. Hence there is a $D \in \operatorname{Diag}(L)$ such that $G \cong \uparrow w_{D}^{\ell}(L) \times \uparrow w_{D}^{r}(L)$. Therefore, part (ii) follows from Lemma 4.9. 
Part (iii) is included in (the last sentence of) [7, Theorem 11]. Finally, the existence in part (iv) follows from Proposition 2.1 and Lemma 6.1(ii), while Lemma 4.1 yields the uniqueness.

Proof of Theorem 3.6. First we deal with the particular case when $L$ is a glued sum indecomposable slim semimodular lattice. Fix a planar diagram $D$ of $L$. Since $S \subseteq\left[0_{S}, 1_{S}\right] \in \mathcal{P}(L)$ holds for all covering squares $S$ of $L$, we conclude that 3.1(i) holds in $\mathcal{P}_{\max }(L)$. So does 3.1 (ii) by Lemma 9.3. To show 3.1(iii), assume that $(I, J) \in \mathcal{E}\left(\mathcal{P}_{\max }(L)\right)$. Then $1 \leq|I \cap J| \leq 2$ by Lemma 9.5. Since 3.1(iii) clearly holds by Lemma 9.4 if $|I \cap J|=1$, we assume that $|I \cap J|=2$. Then $I \cap J$ is of the form $\{x \prec y\}$ since it is a convex sublattice. Lemma (5.5) or Lemma 9.5 yields that $x, y \in \mathcal{B}^{D}(I) \cap \mathcal{B}^{D}(J)$.

Assume for a contradiction that $y \notin\left\{1_{I}, 1_{J}\right\}$. Then $y \in \mathcal{B}_{\text {south }}^{D}(I) \cap \mathcal{B}_{\text {south }}^{D}(J)$ and $x$ belongs to both $\mathcal{B}^{D}(I) \backslash \mathcal{B}_{\text {north }}^{D}(I)$ and $\mathcal{B}^{D}(J) \backslash \mathcal{B}_{\text {north }}^{D}(J)$. Hence, by $(2.14), x$ has a cover $y_{1} \in I \backslash\{y\}$, and it also has a cover $y_{2} \in J \backslash\{y\}$. We have that $y_{1} \in I \backslash J$ and $y_{2} \in J \backslash I$ since $y$ is the largest element of $I \cap J$. Hence $y, y_{1}$ and $y_{2}$ are three distinct covers of $x$, which contradicts (2.1).

Therefore, up to the $I-J$ symmetry, we can assume that $y=1_{J}$. This, $x \prec y$ and $\{x, y\} \subseteq \mathcal{B}^{D}(I) \cap \mathcal{B}^{D}(J)$ imply that $I \cap J=\{x, y\} \subseteq \mathcal{B}_{\text {north }}^{D}(J)$. If we had $y=1_{I}$, then Lemma 9.2 would yield that $I=J$, contradicting $(I, J) \in \mathcal{E}\left(\mathcal{P}_{\max }(L)\right)$. Hence, taking $\{x, y\} \subseteq \mathcal{B}^{D}(I) \cap \mathcal{B}^{D}(J)$ into account, $y$ belongs to $\mathcal{B}^{D}(I) \backslash\left\{1_{I}\right\}=\mathcal{B}_{\text {south }}^{D}(I)$, which gives that $I \cap J=\{x, y\} \subseteq \mathcal{B}_{\text {south }}^{D}(I)$. This proves that $\mathcal{P}_{\max }(L)$ satisfies 3.1(iii). Thus, Theorem 3.6 holds for the slim case.

Next, we drop the assumption that $L$ is slim. Let $L^{\prime}$ be the full slimming sublattice of $L$ with respect to a fixed planar diagram $D$. By Lemma 6.1(iv), $L^{\prime}$ is a glued sum indecomposable slim semimodular lattice. If we consider the intervals $I$ as pairs of elements $\left(0_{I}, 1_{I}\right)$, then $\mathcal{P}_{\max }(L)$ and $\mathcal{P}_{\max }\left(L^{\prime}\right)$ become the same by Lemma 6.1(vii). Hence the already proven slim case of the theorem together with Lemma 6.1(viii) completes the proof.

Proof of Theorem 3.4. We already know from Lemma 8.1 that (ii) $\Leftrightarrow$ (iii) $\Leftrightarrow$ (iv) $\Leftrightarrow(\mathrm{v}) \Leftrightarrow$ (vi). Moreover, (iv) $\Rightarrow$ (vii) follows from Theorem 2.4. Hence it suffices to show that (vii) $\Rightarrow(\mathrm{v}),(\mathrm{v}) \Rightarrow$ (i) and (i) $\Rightarrow$ (iii).

Assume that (vii) holds. Let $F$ be a diagram of the four-element rectangular lattice $S$ from which a diagram $D$ of $L$ is obtained first by adding forks, and then by adding eyes. Then $w_{F}^{\ell}(S)$ is the leftmost coatom of $F, w_{F}^{r}(S)$ is its rightmost coatom, and their intersection is 0 . They remain the leftmost and the rightmost coatoms of the actual diagram, respectively, if we add forks and eyes. Furthermore, the least element of the lattice does not change. Hence $w_{F}^{\ell}(S)$ and $w_{F}^{r}(S)$ will become the leftmost coatom and the rightmost coatom of $D$, and $w_{F}^{\ell}(S) \wedge w_{F}^{r}(S)=$ $0_{S}=0_{L}$. This shows that (vii) $\Rightarrow(\mathrm{v})$ holds.

Assume that $(\mathrm{v})$ holds. Then there exists a diagram $D \in \operatorname{Diag}(L)$ such that for the (unique) coatoms $a \in \mathcal{B}_{\text {left }}^{D}(L)$ and $b \in \mathcal{B}_{\text {right }}^{D}(L)$ we have that $a \wedge b=0$. Notice that $a$ and $b$ are the leftmost coatom and the rightmost coatom with respect to $D$, respectively. Let $\mathcal{H}$ be a patchwork system for $L$. By Proposition 3.2 , it is a patchwork system for the diagram $D$. Let $c$ be the right neighbor of $a$ in $D$; it is a coatom. Let $S=[a \wedge c, a \vee c=1]$; it is a 4-cell of $D$ by [7, Lemma 13]. Hence it is a covering square, and it is a subset of some $I \in \mathcal{H}$ by 3.1(i). Therefore, there is an $I \in \mathcal{H}$ such that $a \in I$ and $1_{I}=1_{L}$. Similarly, there is a $J \in \mathcal{H}$ 
such that $b \in J$ and $1_{J}=1_{L}$. Assume for a contradiction that $I \neq J$. Then $(I, J) \in \mathcal{E}\left(\mathcal{P}_{\max }(L)\right)$ since $1_{L} \in I \cap J$ shows that $I \cap J$ is nonempty. Hence 3.1(iii) yields that $1 \in I \cap J \subseteq \mathcal{B}_{\text {south }}^{D}(I) \cup \mathcal{B}_{\text {south }}^{D}(J)$, which is a contradiction since $1_{K}$ is never on the southern boundary of a rectangular interval $K$. Hence $I=J$. Since $0_{L}=a \wedge b \in I$ and $1_{L} \in I$, we obtain that $\boldsymbol{H}=\{I\}$ by Remark 3.3(i). This proves the implication $(\mathrm{v}) \Rightarrow$ (i).

Next, to show that (i) implies (iii), assume that (iii) fails. We have to show that (i) also fails. We can assume that $L$ is glued sum indecomposable since otherwise (i) fails by definition. Fix a diagram $D \in \operatorname{Diag}(L)$. By the assumption, there are a proper ideal $I$ and a proper filter $F$ such that $L=I \cup F$, and $C:=I \cap F$ is a chain. We assume that $I$ and $F$ are chosen so that $|C|$ is minimal. By (2.6), $I$ and $F$ are planar lattices, and they are clearly semimodular. Let $C=[a, b]=\left[0_{F}, 1_{I}\right]$. We conclude that $a<b$ since otherwise $a=b$ would be comparable with all elements of $L$, contradicting the glued sum indecomposability of $L$. Since $I$ and $F$ are proper subsets, $I \neq C \neq F$ and $|I|,|F| \geq 3$. Assume for a contradiction that there is an $x \in I \backslash\{0, b\}$ such that $I=\downarrow x \cup \uparrow x$. Then $a \in \uparrow x$ would imply that $F \subseteq \uparrow x$, whence $L=\uparrow x \cup \downarrow x$ would contradict the glued sum indecomposability of $L$. Therefore $a \in \downarrow x \backslash \uparrow x$, that is, $a<x<b$. Since $I=\downarrow x \cup \uparrow x=\downarrow x \cup[x, b]$ and $[x, b] \subseteq F$, we can replace $I$ by $\downarrow x$ in the original decomposition. Then $C=[a, b]$ is replaced by $[a, x]$, which contradicts the minimality of $|C|$. Hence there is no $x$ with $I=\downarrow x \cup \uparrow x$. This together with $|I| \geq 3$ implies that $I$ is glued sum indecomposable. We have not used semimodularity, so $F$ is also glued sum indecomposable by duality.

Let $a_{1}$ and $b_{1}$ be the unique elements of $C=[a, b]$ such that $a \prec a_{1}$ and $b_{1} \prec b$. Since $F$ is glued sum indecomposable, $a=0_{F}$ has a cover $a_{2}$ distinct from $a_{1}$. The glued sum indecomposability of $I$ yields that $b=1_{F}$ has a lower cover $b_{2}$ distinct from $b_{1}$. Since $C=I \cap F$ is a chain containing $a_{1}$, we obtain that $a_{2} \notin C$. But $a_{2} \in F$, whence $a_{2} \in F \backslash I$. The dual consideration shows that $b_{2} \in I \backslash F$. If $a$ belonged to $\operatorname{int}^{D}(I)$, then (2.8) together with (2.6) would imply that $a_{2} \in I$, a contradiction. Hence $a \in \mathcal{B}^{D}(I)$. Dually, we obtain that $b \in \mathcal{B}^{D}(F)$. Without loss of generality, we can assume that $a \in \mathcal{B}_{\text {left }}^{D}(I)$. Since $\mathcal{B}_{\text {left }}^{D}(I)$ is a maximal chain in $I$, we have that $\left\{x \in \mathcal{B}_{\text {left }}^{D}(I): a \leq x\right\}$ is a maximal chain in $\left[a, 1_{I}\right]=[a, b]=C$. But $C$ is itself a chain, whence

$$
C=\left\{x \in \mathcal{B}_{\text {left }}^{D}(I): a \leq x\right\} \subseteq \mathcal{B}_{\text {left }}^{D}(I)
$$

Since now we cannot assume that $b \in \mathcal{B}_{\text {right }}^{D}(F)$, lattice duality yields only that $C \subseteq \mathcal{B}_{\text {left }}^{D}(F)$ or $C \subseteq \mathcal{B}_{\text {right }}^{D}(F)$. However, we claim that

$$
C \subseteq \mathcal{B}_{\text {left }}^{D}(I) \cap \mathcal{B}_{\text {right }}^{D}(F)
$$

In view of the previous observation, it suffices to exclude that $C \subseteq \mathcal{B}_{\text {left }}^{D}(F)$. Assume for a contradiction that $C \subseteq \mathcal{B}_{\text {left }}^{D}(F)$, and keep (10.1) in mind. Let $x \in$ $\mathcal{B}_{\text {left }}^{D}(L)$ be the unique element with $h(x)=h(b)$. We obtain from (5.3) that $x$ is on the left of $b$. On the other hand, $x \in I$ or $x \in F$, and $b \in C \subseteq \mathcal{B}_{\text {left }}^{D}(I) \cap \mathcal{B}_{\text {left }}^{D}(F)$. Hence (5.3) (applied to $I$ or $F$ ) yields that $b$ is on the left of $x$. Using (5.2) we conclude that $b=x \in \mathcal{B}_{\text {left }}^{D}(L)$. Hence we obtain from $(5.5)$ that $\mathcal{B}_{\text {left }}^{D}(I) \subseteq \mathcal{B}_{\text {left }}^{D}(L)$. Let $E_{1}:=E \cap \uparrow b$. It is a maximal chain in $\uparrow b$. Therefore, since $\mathcal{B}_{\text {left }}^{D}(I)$ is a maximal chain in $\downarrow b=I$, we obtain that $E:=\mathcal{B}_{\text {left }}^{D}(I) \cup E_{1}$ equals $\mathcal{B}_{\text {left }}^{D}(L)$. Notice that $E$ is a maximal chain in $L$. Let $W:=\mathcal{B}_{\text {right }}(I) \cup E_{1}$; it is also a maximal chain of $L$. 
Let $y \in \mathcal{B}_{\text {right }}^{D}(I)$ denote the unique element with $h(y)=h(a)$. Since $a \in \mathcal{B}_{\text {left }}(I) \backslash$ $\left\{0_{I}, 1_{I}\right\}$ and $I$ is glued sum indecomposable, $a \notin \mathcal{B}_{\text {right }}^{D}(I)$. Hence (5.3) yields that $a$ is strictly on the left of $y$. So we obtain from (5.1) that

$a$ is strictly on the left of $W$.

Trivially (or it follows from (5.1) and (5.3)), we have that $a_{2}$ is on the right of $E=\mathcal{B}_{\text {left }}^{D}(L)$. If $a_{2}$ was on the left of $W$, then (5.6), applied for $(0, b)$ instead of $(u, v)$, would imply that $a_{2} \in I$, which contradicts $a_{2} \in F \backslash I$. Therefore, $a_{2}$ is strictly on the right of $W$. This together with (10.3) and $a \prec a_{2}$ contradicts (2.5). Thus, (10.2) is proved.

The restriction of $D$ to $I$ and $F$ will be denoted by $D_{I}$ and $D_{F}$, respectively. By Theorem 3.6 and Proposition 3.2, $\mathcal{P}_{\max }(I)$ and $\mathcal{P}_{\max }(F)$ are patchwork systems for $D_{I}$ and $D_{F}$, respectively. Let $\mathcal{H}:=\mathcal{P}_{\max }(I) \cup \mathcal{P}_{\max }(F)$; we claim that it is a patchwork system for $D$.

Assume for a contradiction that there is a covering square $S=\{u \wedge v, u, v, u \vee v\}$ such that $S \nsubseteq I$ and $S \nsubseteq F$. Then, say, $u \in F \backslash I$ and $v \in I \backslash F$. Extend $C$ to a maximal chain $C^{\bullet}$ of $L$. Clearly, $u \vee v \in F \backslash I$. Since $h(a)=h\left(0_{F}\right) \leq$ $h(u)=h(v) \leq h\left(1_{I}\right)=h(b)$, the chain $C=[a, b]$ has a unique element $x$ such that $h(x)=h(u)=h(v)$. Furthermore, $v \notin F$ gives that $v<b$, whence $h(v)+1 \leq h(b)$. Consequently, $h(u \vee v)=h(v)+1 \leq h(b)$, and there is an element $y \in \bar{C}$ with $h(y)=h(u \vee v)$.

Using that $v \in I$ and $x \in C \subseteq \mathcal{B}_{\text {left }}(I)$, (5.3) yields that $v$ is on the right of $x \in C^{\bullet}$. This fact, $v \neq x$ and (5.1) yield that $v$ is strictly on the right of $C^{\bullet}$. Since $u \vee v \neq y \in C \subseteq \mathcal{B}_{\text {right }}^{D}(F)$, the left-right dual of (5.3) yields that $u \vee v$ is strictly on the left of $y$. Hence (5.1) yields that $u \vee v$ is strictly on the left of $C \bullet$. Thus, $v \prec u \vee v$ contradicts (2.5). This proves that each covering square is either a subset of $I$ or a subset of $F$. This implies that 3.1(i) holds for $\mathcal{H}$.

Next, assume that $(J, K) \in \mathcal{E}(\boldsymbol{H})$. If $J, K \in \mathcal{P}_{\max }(I)$ or $J, K \in \mathcal{P}_{\max }(F)$, then 3.1(ii) and 3.1(iii) clearly hold for $(J, K)$. Hence we can also assume that $J \in \mathcal{P}_{\max }(I)$ and $K \in \mathcal{P}_{\max }(F)$. Since $J \cap K \subseteq I \cap F=C$ and $C$ is a chain, 3.1(ii) holds for $(J, K)$.

Using that $\operatorname{int}^{D}(J) \subseteq \operatorname{int}^{D}(I)$ by (2.9) and $C \subseteq \mathcal{B}^{D}(I)$ by (10.2), we obtain that $\operatorname{int}^{D}(J) \cap C=\emptyset$. Hence $\left.J \cap C=\operatorname{int}^{D}(J) \cap C\right) \cup\left(\mathcal{B}^{D}(J) \cap C\right)=\mathcal{B}^{D}(J) \cap C \subseteq \mathcal{B}^{D}(J)$. Assume for a contradiction that $J \cap C \nsubseteq \operatorname{Mi}(J)$. Then there is an $x \in J \cap C$ with at least two covers in $J$. All these covers belong to $C$ since $F$ is a filter. This is a contradiction since $C$ is chain. Consequently, $J \cap C \subseteq \operatorname{Mi}(J)$. Combining this with $J \cap C \subseteq \mathcal{B}^{D}(J)$ and (2.14), we obtain that $J \cap C \subseteq \mathcal{B}_{\text {north }}^{D}(J)$. This together with $J \cap K \subseteq C$ yields that $J \cap K \subseteq J \cap K \cap C \subseteq \bar{J} \cap C \subseteq \mathcal{B}_{\text {north }}^{D}(J)$. Dualizing the above argument (in particular, replacing (2.14) by (2.15)) we obtain that $J \cap K \subseteq J \cap K \cap C \subseteq K \cap C \subseteq \mathcal{B}_{\text {south }}^{D}(K)$. Hence 3.1(iii) (with $D$ ) holds. Therefore, $\mathcal{H}$ is a patchwork system for $D$. Since $|\boldsymbol{H}|=\left|\boldsymbol{P}_{\max }(I) \cup \boldsymbol{P}_{\max }(F)\right|=$ $\left|\mathcal{P}_{\max }(I)\right|+\left|\mathcal{P}_{\max }(F)\right| \geq 1+1=2$, we conclude that (i) fails. This completes the proof of the implication (i) $\Rightarrow$ (iii).

Proof of Corollary 3.5. By Herrmann [20] or [3, Lemma 6.1], the Hall-Dilworth gluing (not only over chains) preserves semimodularity. By finiteness, the rest of the statement follows from (iii) $\Leftrightarrow$ (iv) of Theorem 3.4. 
Proof of Corollary 3.8. As mentioned right before Corollary 3.7, only the second part needs a proof. Since non-chain intervals of length 2 are atomistic, all we have to show is that if $I$ is an interval of length greater than 2, then $I$ is not atomistic. Assume the contrary, and let $\left\{a_{1}, \ldots, a_{n}\right\}$ be a maximal independent system of atoms of $I$. Then $n$ is the length of $I$ and these atoms generate a Boolean sublattice $B$ of length $n$, see Grätzer [12, Theorem IV.2.5] or [13, Theorem 381]. This is a contradiction since $B$ is not planar for $n \geq 3$.

\section{REFERENCES}

[1] Czédli, G.: Factor lattices by tolerances. Acta Sci. Math. (Szeged) 44 (1982), 35-42

[2] Czédli, G.: The matrix of a slim semimodular lattice. Order (in press)

[3] Czédli, G.: Representing homomorphisms of distributive lattices as restrictions of congruences of rectangular lattices. Algebra Universalis (submitted)

[4] Czédli, G., Schmidt, E.T.: How to derive finite semimodular lattices from distributive lattices? Acta Mathematica Hungarica 121, 277-282 (2008)

[5] Czédli, G., Schmidt, E. T.: Some results on semimodular lattices, Contributions to General Algebra 19 (Proc. Olomouc Conf. 2010), Johannes Hein verlag, Klagenfurt (2010), 45-56. ISBN 978-3-7084-0407-3

[6] Czédli, G., Schmidt, E.T.: The Jordan-Hölder theorem with uniqueness for groups and semimodular lattices. Algebra Universalis (in press)

[7] Czédli, G., Schmidt, E.T.: Slim semimodular lattices. I. A visual approach, Order (to appear), DOI: $10.1007 /$ s11083-011-9215-3, see http://www.math.u-szeged.hu/ czedli/

[8] Czédli, G., Schmidt, E.T.: Intersections of composition series in groups and slim semimodular lattices by permutations, submitted

[9] Czédli, G., Ozsvárt, L., Udvari, B.: How many ways can two composition series intersect? Discrete Mathematics (submitted)

[10] Day, A., Herrmann, C.: Gluings of modular lattices. Order 5 (1988), 85-101

[11] Dilworth, R.P.: A decomposition theorem for partially ordered sets. Ann. of Math. 51 (1951), 161-166

[12] Grätzer, G.: General Lattice Theory, 2nd edn. Birkhäuser Verlag, Basel (1998)

[13] Grätzer, G.: Lattice Theory: Foundation. Birkhäuser Verlag, Basel (2010) xxix+613 pp. ISBN: 978-3-0348-0017-4

[14] Grätzer, G.: The congruences of a finite lattice. A proof-by-picture approach. Birkhäuser Verlag, Boston, (2006)

[15] Grätzer, G., Knapp, E.: Notes on planar semimodular lattices. I. Construction. Acta Sci. Math. (Szeged), 73 (2007), 445-462

[16] Grätzer, G., Knapp, E.: Notes on planar semimodular lattices. III. Congruences of rectangular lattices. Acta Sci. Math. (Szeged), 75 (2009), 29-48

[17] Grätzer, G., Knapp, E.: Notes on planar semimodular lattices. IV. The size of a minimal congruence lattice representation with rectangular lattices. Acta Sci. Math. (Szeged), 76 (2010), 3-26

[18] Grätzer, G., Nation, J.B.: A new look at the Jordan-Hölder theorem for semimodular lattices. Algebra Universalis 64 (2010), 309-311

[19] Grätzer, G., Wares, T.: Notes on planar semimodular lattices. V. Cover-preserving embeddings of finite semimodular lattices into simple semimodular lattices. Acta Sci. Math. (Szeged), 76 (2010), 27-33

[20] Herrmann, C.: S-verklebte Summen von Verbänden. Math. Z. 130 (1973), 255-274.

[21] Hölder, O.: Zurückführung einer beliebigen algebraischen Gleichung auf eine Kette von Gleichungen. Math. Ann. 34 (1889), 26-56

[22] Jordan, C.: Traité des substitutions et des équations algebraique. Gauthier-Villars (1870)

[23] Kelly, D., Rival, I.: Planar lattices. Canad. J. Math. 27 (1975), 636-665

[24] Schmidt, E.T.: Congruence lattices and cover preserving embeddings of finite length semimodular lattices. I. Acta Sci. Math. Szeged (in press)

[25] Stern, M.: Semimodular Lattices. Theory and Applications. Encyclopedia of Mathematics and its Applications, 73. Cambridge University Press (1999) 
E-mail address: czedli@math.u-szeged.hu

URL: http://www.math.u-szeged.hu/ czedli/

University of Szeged, Bolyai Institute, Szeged, Aradi vértanúk tere 1, Hungary 6720

E-mail address: schmidt@math.bme.hu

URL: http://www.math.bme.hu/ schmidt/

Mathematical Institute of the Budapest University of Technology and Economics, Mǘggyetem RKP. 3, H-1521 Budapest, Hungary 